\begin{tabular}{|l|l|l|l|l|l|l|l|l|l|l|l|}
\hline \multirow{2}{*}{ SPi } & \multicolumn{4}{|l|}{ Journal Code: } & \multicolumn{4}{|l|}{ Article ID } & Dispatch: 25.11 .14 & CE: Shinar Q. Ragudo \\
\cline { 2 - 8 } & N & A & G & & 2 & 3 & 4 & 4 & No. of Pages: 24 & ME: \\
\hline
\end{tabular}

\title{
Numerical simulation of percussive drilling
}

\author{
Alexandre Depouhon ${ }^{1,2, *, \dagger}$, Vincent Denoël ${ }^{1}$ and Emmanuel Detournay ${ }^{2}$ \\ ${ }^{1}$ Structural and Stochastic Dynamics, ArGEnCo, Université de Liège, Belgium \\ ${ }^{2}$ Department of Civil, Environmental, and Geo-engineering, University of Minnesota, MN, U.S.A.
}

\begin{abstract}
SUMMARY
This paper presents a novel dynamical model to analyze the long-term response of a percussive drilling system. This departs from existing approaches that usually consider a single activation and bit/rock interaction cycle for the analysis of the process performance. The proposed model integrates the axial dynamics of an elastic piston and an elastic drill bit, a motion-dependent pressure law to drive the piston, and a generalized bit/rock interaction law representative of the dynamic indentation taking place at the bit/rock interface. It applies to down-the-hole percussive drilling as well as top-hole, with minor modifications. The model does not account for the angular motion or the hole cleaning, however. The model is first formulated mathematically; then, a finite-dimensional approximation is proposed for computations. Numerical analyses of the model response, for a low-size down-the-hole percussive system, follow. The period-1 stationary response for the reference configuration is studied in detail, and parametric analyses assessing the influence on the rate of penetration of the bit/rock interaction parameters, the feed force, and the percussive activation parameters are conducted. These analyses reveal that the multiscale nature of the process is well captured by the model and recover expected trends for the influence of the parameters. They also suggest that a significant increase of the penetration rate can be achieved by increasing the percussive frequency. Copyright ( $) 2014$ John Wiley \& Sons, Ltd.
\end{abstract}

Received 1 September 2014; Revised 7 November 2014; Accepted 13 November 2014

KEY WORDS: percussive drilling; dynamical systems; bit/rock interaction; rate of penetration

\section{INTRODUCTION}

Percussive drilling has a long history. Appearing in China for water-well drilling around 2000 BC [1], the technology is, nowadays, widely used in the Earth's resources industries to drill hard rock. It notably finds applications in mining (exploration, drill, and blast), water well and geothermal drilling, and shallow oil and gas exploration and production. The principle of percussive drilling is simple: via a repeated collision mechanism between two elastic bodies, impulsive loads are sent down to the bit, which penetrates the rock by crushing and chipping [2] as a result of dynamic indentation. Although several implementations of the technology have seen the day, two dominate the drilling landscape: top-hole $(\mathrm{TH})$ and down-the-hole (DTH) percussive drills. In the former realization, the percussion takes place on the surface, and the stress pulses generated by the percussive activation travel to the bit via the drill pipes or the drill steel. In the latter version, the percussive activation is performed down the hole by a moving piston directly impacting the bit.

Given the complexity of the drilling process, theoretical developments lag well behind the knowhow and experience that have been gathered over years of field practice, however [3]. In particular, the scientific community is yet to provide an integrated model of percussive drilling that couples the process components and analyzes its performance from a dynamical system standpoint.

\footnotetext{
*Correspondence to: Alexandre Depouhon, Structural and Stochastic Dynamics, ArGEnCo, Université de Liège, Belgium.

${ }^{\dagger}$ E-mail: depou002@umn.edu
} 
The availability of such a model—provided it is reliable — could dramatically influence the development of the technology and contribute to the-closed-loop control so as to optimize the drilling process. For instance, the objective of a feedback controller could be to maximize the average rate of penetration, noting that the existence of an optimum rate of penetration-the so-called sweet spot-has been observed in the field [4].

So far, two main directions have been followed as to the modeling of percussive drilling and the assessment of its efficiency or performance. Both present limitations, however.

The first is based on the analysis of energy transfers between the piston and the rock penetration process and the evaluation of how much penetration is achieved for a single percussive activation. Closed-form expressions for the drilling efficiency, based on wave propagation considerations and a bilinear bit/rock interaction model, were first proposed in [2, 5-7], on the assumption of contact closure at the arrival of the incoming stress wave at the bit/rock interface, whose form was arbitrarily chosen. Numerical investigations then followed, enabling the relaxation of some of the assumptions behind the analytical models. For instance, Lundberg [8] relaxed the arbitrariness of the incoming stress wave by accounting for the geometries of the piston and the bit_while Chiang and Elías [9] explicitly modeled the bit/rock interaction using finite elements. Additional results for the single activation analysis can be found in [10-12]. Despite the insight these analyses provide, it is questionable as to what extent such results can be extrapolated to the dynamical setting of successive percussive activations. Drilling systems can experience a variety of stationary responses. That the bit be at rest at the occurrence of percussive activation is only representative of one them, provided the system angular dynamics is not accounted for.

The second modeling direction is that of the drifting oscillator initiated at the University of Aberdeen; it is based on a reduction of the drilling system to a rigid bit unilaterally constrained by a sliding interface, representative of the bit/rock interaction, and subjected to harmonic loading. Two families of interface laws have been investigated: variations on the serial connection between a plastic slider and a viscoelastic cell [13], and piecewise power laws [14] that generalize, in a sense, the bilinear force/penetration model commonly used to represent bit/rock interaction in percussive drilling [15]. Such systems have been demonstrated to exhibit optimal drilling configurations; that is, sets of control parameters for which the average rate of penetration can be maximized. However, their direct applicability to percussive drilling is open to questions. The first family of interface laws is not in line with the experimental evidence on bit/rock interaction (for instance, the model allows unbounded penetration under a static load above the slider threshold), while harmonic excitation is not representative of the impulsive loading generated by the percussive activation.

As a first step in formulating a comprehensive integrated dynamical model of percussive drilling, this paper proposes a novel model of the process that couples the piston and bit axial dynamics to a bilinear bit/rock interaction law generalized to the case of multiple impacts and a simplified pressure law to represent the piston activation. In light of the available knowledge, wave propagation appears to play an essential role in the process dynamics, notably on the contact interaction between the piston and the bit; the two bodies are therefore considered as elastic. Inherent to the formulation is thus the account for the multiple timescales on which the problem evolves. While the focus of this research is on DTH percussive drilling, the model that has been developed could as well be used to describe TH percussive drilling, with minor adjustments.

The combination of a finite element discretization in space with an event-driven time integration strategy is proposed to solve the piecewise partial differential equations that govern the process model. The versatility of this approach, developed by the authors in the case of unilateral elastic constraints [16], makes it possible to relax some of the present model simplifications in future work, for example, the replacement of the pressure law by a thermodynamical model [17] or the explicit modeling of the bit/rock interaction [18, 19] rather than the simple force/penetration relationship we have assumed. The proposed model and simulation procedure are thus expected to serve as a stepping stone toward a more integrated analysis of percussive drilling.

The paper is organized as follows. Section 2 is dedicated to the model formulation, with the establishment of the partial differential equations governing the model dynamics. In Section 3, the computational version of the model is given; that is, details about the numerical procedure to integrate the governing equations are given. Numerical results are then presented in Section 4; the focus 
is set on (i) the stationary response predicted by the model at a reference configuration corresponding to a low-size DTH apparatus and (ii) on the influence of the model parameters on the predicted average rate of penetration around that reference point. A synthesis of our findings is proposed in the last section.

\section{DOWN-THE-HOLE PERCUSSIVE DRILLING MODEL}

A DTH hammer system consists of three major parts: (i) the drill bit, (ii) the piston, and (iii) the hammer casing. As the yanguard of the drilling system, the drill bit penetrates into the rock medium under the percussive activation delivered by the pneumatically activated piston that repeatedly impacts it. The bit is at the same time subjected to an angular motion and a vertical force transmitted by the casing that connects the hammer to the drill string, thereby establishing the link with the surface rig.

The model presented here is based on a simplification of the drilling apparatus to two of its base components, namely an elastic piston interacting with an elastic bit, whose penetration into the rock is represented by a bit/rock interaction law. Figure 1 illustrates the considered geometry, with the steel elastic bodies reduced to collinear cylinders of identical cross section, and the applied external forcing of gravity $G$, air pressure on the piston $F_{A}$, and feed force on the bit $F_{S}$. This model builds on two essential ingredients for the representation of the process: (i) a dynamical model of the mechanical system that properly captures the piston/bit interaction as governed by the interplay of waves propagating in the system and (ii) a generalized bit/rock interaction law representative of the dynamic indentation taking place during drilling. Angular motion of the system and wave propagation in the hammer casing and drill string are neglected.

\subsection{Equations of motion}

Considering the reduction of the piston and the bit to one-dimensional (1D) bodies, an approximation justified by their slenderness and concurring with the analyses on the limited three-dimensional effects on the response of DTH systems $[9,11]$, the motion of the piston and the bit can be described by the scalar wave equation that expresses the balance of linear momentum of an infinitesimal slice of material [20-22].

Let $O \boldsymbol{e}_{x}$ be the global axis system, with respect to which positions and displacements are measured, and $O_{i} \boldsymbol{e}_{i}, i \in\{p ; b\}$ be local axis systems moving with the top surface of the piston (subscript $p$ ) and the bit (subscript $b$ ), respectively. Following these definitions, $x_{i}\left(X_{i}, t\right)$, the position of a material point in the global axis system at time $t$, can be expressed as the sum of the position of the local axis system origin $x_{i, 0}$ at the origin of time, its associated Lagrangian coordinate $X_{i} \in\left[0, L_{i}\right]$, and the displacement $u_{i}\left(X_{i}, t\right)$ :

$$
x_{i}\left(X_{i}, t\right)=x_{i, 0}+X_{i}+u_{i}\left(X_{i}, t\right) .
$$

Upon assuming that the initial displacement field is zero, $u_{i}\left(X_{i}, 0\right)=0$, this relationship verifies

$$
x_{i}\left(X_{i}, 0\right)=x_{i, 0}+X_{i} .
$$

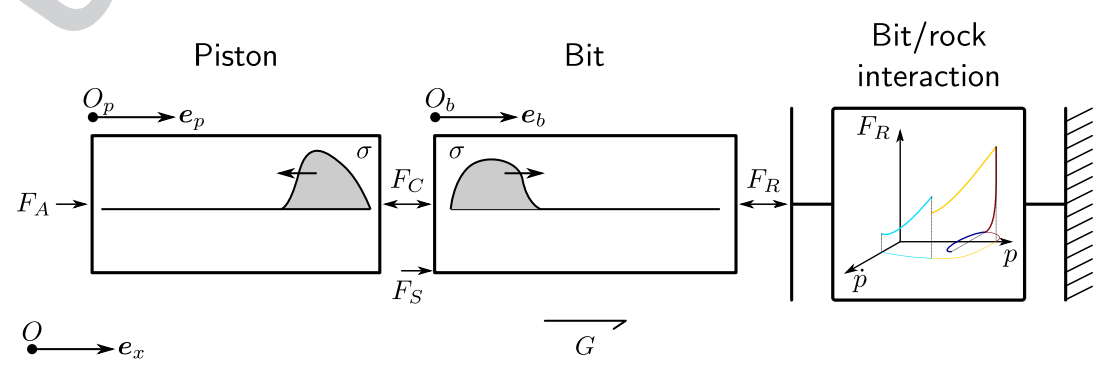

Figure 1. Simplified representation of a down-the-hole percussive drilling system. 
Under this notation, the generic wave equation ruling the motion of the piston and the bit reads

$$
\rho A \frac{\partial^{2} u_{i}}{\partial t^{2}}\left(X_{i}, t\right)=E A \frac{\partial^{2} u_{i}}{\partial X_{i}^{2}}\left(X_{i}, t\right)+\rho A f_{i}
$$

Dimensional parameters $\rho$ and $E$ refer to the steel density and Young's modulus; the cross section of the cylinders is denoted by $A$ and the external body forcing term by $f_{i}$, which depends on the considered body. Coupling between the motion of each body, as well as with the hole bottom, enters the equations of motion through the boundary conditions.

Specializing to the piston, the boundary conditions at the top and bottom ends are given by

$$
\frac{\partial u_{p}}{\partial X_{p}}(0, t)=0, \quad 0 \leq-\frac{\partial u_{p}}{\partial X_{p}}\left(L_{p}, t\right) \perp g(t) \geq 0,
$$

where the complementarity condition expresses the Signorini condition [23] at the contact interface. Either it is open with a positive gap function, the distance between the piston bottom end and the bit top end,

$$
\begin{aligned}
g(t) & =x_{b}(0, t)-x_{p}\left(L_{p}, t\right) \\
& =g_{0}+u_{b}(0, t)-u_{p}\left(L_{p}, t\right)
\end{aligned}
$$

with $g_{0}$ denoting the initial gap, and a zero contact stress $E \partial u_{p} / \partial X_{p}\left(L_{p}, t\right)=0$. Or it is closed with a zero gap function $g(t)=0$ and a compressive contact stress $E \partial u_{p} / \partial X_{p}\left(L_{p}, t\right) \leq 0$. The body force includes the action of gravity $G$ and that of the pressure force $F_{A}$ that we choose to apply as a body force so as to not generate spurious wave fronts through its piecewise constant definition

$$
f_{p}=G+\frac{F_{A}}{\rho A L_{p}} .
$$

As to the bit, its boundary conditions read

$$
0 \leq-\frac{\partial u_{b}}{\partial X_{b}}(0, t) \perp g(t) \geq 0, \quad-E A \frac{\partial u_{b}}{\partial X_{b}}\left(L_{b}, t\right)=F_{R},
$$

where $F_{R}$ denotes the bit/rock interaction force. The body force includes gravity and the feed force applied from the surface $F_{S}$ that we also define as a body force to prevent spurious wave fronts, which would result from its application at a single point

$$
f_{b}=G+\frac{F_{S}}{\rho A L_{b}} .
$$

\subsection{Pressure force on piston}

Pneumatic hammers usually comprise two pressure chambers that are alternatively fed with compressed air. In valveless systems, the access of the air flow to either chamber follows from the piston motion, more specifically from its position relative to that of the hammer casing. The pressure force applied to the piston thus results from the thermodynamics of the air flow as ruled by the motion of the piston [17].

Rather than invoking a thermodynamical model that would provide an accurate definition of the pressure force throughout the piston motion cycle, see [24, Figure 3], we have recourse to a simplified law, based on two parameters only, whose calibration guarantees the orders of magnitude of the percussive frequency, of the impact velocity, and of the piston maximal stroke. The law is based on the relative average motion of the piston/bit system that is given by

$$
u_{r}=\left\langle u_{p}\right\rangle_{X}-\left\langle u_{b}\right\rangle_{X}-g_{0}, \quad v_{r}=\left\langle v_{p}\right\rangle_{X}-\left\langle v_{b}\right\rangle_{X},
$$


with the spatially averaged fields defined by

$$
\left\langle u_{i}\right\rangle_{X}=\int_{0}^{L_{i}} u_{i}\left(X_{i}, t\right) \mathrm{d} X_{i}, \quad\left\langle v_{i}\right\rangle_{X}=\int_{0}^{L_{i}} v_{i}\left(X_{i}, t\right) \mathrm{d} X_{i}, \quad i \in\{p, b\} .
$$

It reads

$$
F_{A}\left(u_{r}, v_{r}\right)= \begin{cases}F_{0} & \text { if }\left(u_{r}, v_{r}\right) \in\left(-\infty,-D_{1}\right] \times \mathbb{R} \cup\left(-D_{1}, 0\right] \times \mathbb{R}^{+} \\ -F_{0} & \text { otherwise. }\end{cases}
$$

Figure 2 illustrates the piecewise definition of the pressure law in the phase plane $\left(u_{r}, v_{r}\right)$ and shows the typical trajectory that would follow a rigid piston in case the bit is at rest; that is, relative motion is absolute. Starting from point 0 with a negative velocity $v_{r, 0}$, the pressure force is defined upwards and the piston accelerated in the same direction. At point 1, the direction of the pressure force switches to downwards; the piston experiences constant deceleration, and its velocity is zero when it reaches point 2. At that point, it starts its descent toward the bit, where it collides upon reaching the contact interface at point 3 . As soon as contact is established at the piston/bit interface, the pressure force direction is set to upwards.

Following the assumptions of rigid piston and bit at rest, the impact velocity at point $3, v_{r, 3}$, the maximal stroke at point $2,-u_{r, 2}$, and the piston free flight(FF) duration $t_{3}$ can be estimated as a function of the law parameters $F_{0}$ and $D_{1}$; they read

$$
\begin{aligned}
v_{r, 3} & =\sqrt{\frac{F_{0} D_{1}}{M_{p}}} \sqrt{4+2 \mu}, \\
-u_{r, 2} & =D_{1}(2+\mu), \\
t_{3} & =\sqrt{\frac{D_{1} M_{p}}{F_{0}}}(\sqrt{4+2 \mu}+2 \sqrt{2 \mu+2}-\sqrt{2 \mu}),
\end{aligned}
$$

where $\mu:=M_{p} v_{r, 0}^{2} / 2 F_{0} D_{1}$ is the ratio between the piston initial kinetic energy $M_{p} v_{r, 0}^{2} / 2$ and the energy provided to the piston over a pressure cycle $E_{P}=F_{0} D_{1}$. Therefore, given reference values for the piston mass $M_{p}$ and the rebound velocity $v_{r, 0}$, parameters $F_{0}$ and $D_{1}$ can be estimated by solving a constrained nonlinear algebraic problem that enforces equality constraints on variables $v_{r, 3}$ and $t_{3}$ and the inequalities $0<D_{1}<-u_{r, 2}$. For the reference configuration given in Table II, we have $M_{p}=7555 \mathrm{~g}$. Setting $v_{r, 3}=8.5 \mathrm{~mm} / \mathrm{ms}, v_{r, 0}=-3 \mathrm{~mm} / \mathrm{ms}, t_{3}=45 \mathrm{~ms}(\equiv 22.2 \mathrm{~Hz})$ and $-u_{r, 2}=100 \mathrm{~mm}$, we obtain $D_{1}=39 \mathrm{~mm}$ and $F_{0}=3064 \mathrm{~N}$, which we round to $D_{1}=$ $40 \mathrm{~mm}$, and $F_{0}=3000 \mathrm{~N}$ for the reference configuration. In addition, for this reference setting,

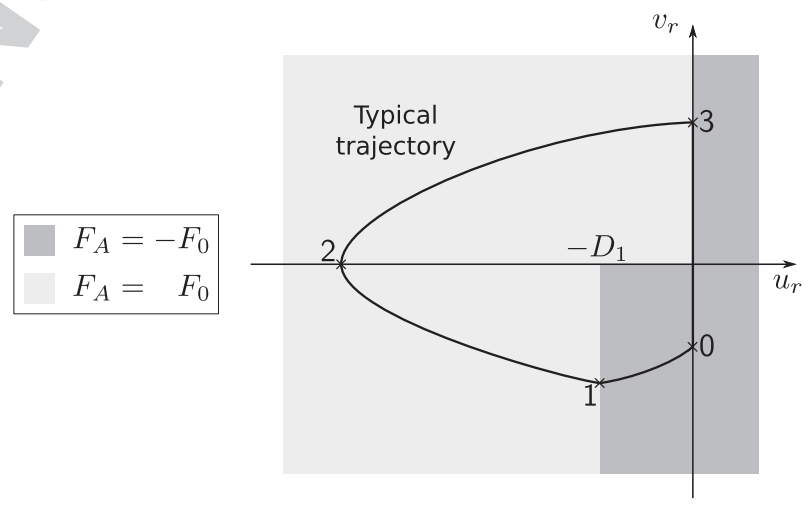

Figure 2. Piecewise-constant pressure model to represent the pneumatic driving of the piston. 
$\mu=0.284=O\left(10^{-1}\right)$. Accordingly, equations (12) naturally reveal the scales intrinsic to the motion of the piston, namely

$$
L_{P}=D_{1}, \quad T_{P}=\sqrt{\frac{D_{1} M_{p}}{F_{0}}}, \quad V_{P}=\sqrt{\frac{F_{0} D_{1}}{M_{p}}},
$$

for the dimensions of length, time, and velocity, respectively. Additionally, the power reference scale associated with the pressure force can be derived; it reads

$$
P_{P}=\sqrt{\frac{F_{0}^{3} D_{1}}{M_{p}}}
$$

Its expression proves useful to analyze the influence of the percussive activation on the model response. Indeed, keeping $P_{P}$ constant, the reference time and velocity scales can be expressed as a function of $D_{1}$ only, yielding the following laws:

$$
T_{P}^{*} \sim D_{1}^{2 / 3}, \quad V_{P}^{*} \sim D_{1}^{1 / 3} .
$$

Under the assumption of constant power scale, the reduction of parameter $D_{1}$ thus leads to that of the period of percussive activation (increase of the percussive frequency) and that of the piston impact velocity at the onset of percussive activation.

Given the piecewise-constant definition of the pressure force, the body-forcing term acting on the piston (6) can be restated as

$$
f_{p}=G+\alpha \frac{F_{0}}{\rho A L_{p}},
$$

with parameter $\alpha \in\{-1 ; 1\}$ defined in accordance with the relative motion.

\subsection{Bit/rock interaction law}

A common representation of bit/rock interaction in percussive drilling is by means of a bilinear law that relates the force on bit $F_{R}$ to the bit penetration $p$; that is, $F_{R}=F_{R}(p)$. Indeed, such phenomenological relation has been shown to be a good approximation to the experimentally and numerically demonstrated characteristics of bit/rock interaction in percussive drilling $\overline{\mathbf{\alpha}}_{\mathbf{2}}(\mathrm{i})$ The force/penetration response consists of two successive phases, one associated with the loading (downward motion) and the other with the unloading (upward motion) of the bit/rock interface $[2,25]$. (ii) This law is rate-independent; that is, it does not significantly depend on the penetration velocity of the indenter in the range of velocities spanned in percussive drilling [26, 27]. (iii) The law can be approximated by a bilinear model that is characterized by two stiffness parameters $[9$, $15,19,28]$, one for the loading phase and one for the unloading phase, $K_{R}$ and $\gamma K_{R}$, respectively. See the experimental and numerical examples reported in Figure 3.

While there is a one-to-one correspondence between bit penetration and displacement for the first drilling cycle, this no longer holds when successive drilling cycles are considered. To generalize the bilinear interaction law to successive interaction cycles, we have followed two steps [31]. This generalization is yet to be validated experimentally.

First, we have introduced the concept of penetration while drilling that relates the current position of the bit $x_{b}(t)=x\left(L_{b}, t\right)$ to the penetration $p^{(n)}(t)$ relative to the $n^{\text {th }}$ (current) drilling cycle by introducing the internal variable $x_{R}^{(n)}$ associated with the position of the rock surface

$$
p^{(n)}(t)=x_{b}(t)-x_{R}^{(n)} .
$$

The internal (history) variable $x_{R}^{(n)}$ is constant over a drilling cycle. It is updated in a stepwise manner at the start of a new drilling cycle so as to guarantee the continuity of the interaction force 


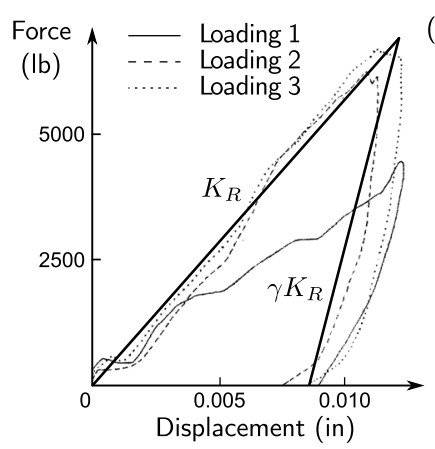

(a) Force

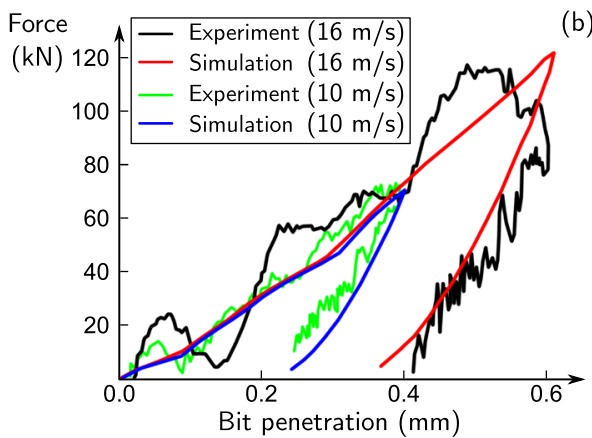

(b)

Figure 3. (a) Experimental force/penetration curves corresponding to the dynamic indentation of Indiana limestone with a chisel bit: the first loading corresponds to impacting the polished rock surface, and successive impacts are on the cleaned but damaged surface. Adapted from [29]. (b) Experimental and numerical force/penetration curves for the dynamic indentation of Kuru granite using a triple-button bit. Adapted from [30].

$F_{R}$ at this transition; that is, at time instants $t_{n}$ that correspond to the closure of the bit/rock interface or a change of the direction of motion of the bit during an unloading phase, its update is given by

$$
x_{R}^{(n+1)}=x_{R}^{(n)}+\Delta x_{R}\left(t_{n+1}\right),
$$

with

$$
\Delta x_{R}\left(t_{n+1}\right)=p^{(n)}\left(t_{n+1}^{-}\right)-\frac{F_{R}\left(t_{n+1}\right)}{K_{R}}
$$

and $p^{(n)}\left(t_{n+1}^{-}\right)=\lim _{\epsilon \rightarrow 0^{+}} p^{(n)}\left(t_{n+1}-\epsilon\right)$. Following this definition, the penetration while drilling is thus defined as the advance of the bit with respect to its position at the start of a drilling cycle; plus, a residual penetration has the previous cycle not completed. Assuming a bilinear interaction law, it then ensues that when the bit/rock interface is closed, the interaction force is given by

$$
F_{R}(t)=K_{R} p^{(n)}(t)
$$

during loading (downward motion) and by

$$
F_{R}(t)=\gamma K_{R}\left(p^{(n)}(t)-p_{u}^{(n)}\right)
$$

during unloading (upward motion). The internal variable $p_{u}^{(n)}$ corresponds to the penetration while drilling at which the interaction force would vanish during the unloading phase. It is defined by

$$
p_{u}^{(n)}=\frac{\gamma-1}{\gamma}\left(x_{b}\left(t_{p}\right)-x_{R}^{(n)}\right)
$$

where $t_{p}$ is the time of peak penetration, that is, the instant at which the bit has a vanishing velocity and the bit/rock interface switches from loading to unloading. The upper penetration implicitly defines the associated upper bit position at which the interaction force would vanish:

$$
x_{b, u}^{(n)}=p_{u}^{(n)}+x_{R}^{(n)} .
$$

Figure 4 illustrates the definitions of the penetration while drilling and the internal variables. At point $\mathrm{A}$, the bit/rock interface closes and drilling cycle 1 starts; $x_{R}^{(1)}$ is set to the bit position. At point $\mathrm{B}$, peak penetration is reached. The unloading phase starts, and $x_{b, u}^{(1)}$ is set. The bit velocity vanishes at point $\mathrm{C}^{-}$, leading to the start of a new drilling cycle. Accordingly, $x_{R}^{(2)}$ is defined in $\mathrm{C}^{+}$using (19); as the interaction force is positive at the transition, it does not correspond to the 

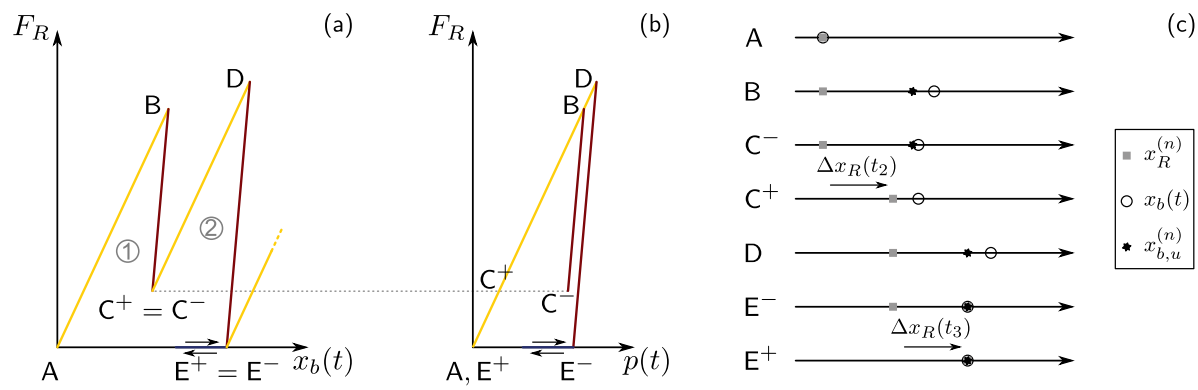

Figure 4. Illustration of the definitions of the penetration while drilling and the internal variables on the basis of the force/position response shown in (a); plot (b) shows the mapping of the trajectory in the force/penetration plane, while schematic diagram (c) represents the update of the internal variables.

physical position of the rock at the start of the drilling cycle, which is given by the position of the bit bottom end $x_{b}\left(t_{2}\right)$. Peak penetration is reached at point $\mathrm{D}$, yielding the definition of the upper position $x_{b, u}^{(2)}$, which the bit attains at $\mathrm{E}^{-}$; this corresponds to the opening of the bit/rock interface and the vanishing of the interaction force. After a period of $F F$, during which the bit/rock interface is open, the interface closes again at point $\mathrm{E}^{+}$where a third drilling cycle starts; consequently, $x_{R}^{(2)}$ is incremented to define $x_{R}^{(3)}$, which, in the absence of a residual force, reflects the position of the rock surface at the start of the drilling cycle.

Second, we have introduced an energy barrier in the interaction model to dissociate static indentation from dynamic indentation. Under this hypothesis, penetration at the start of a drilling cycle is only allowed if the bit has sufficient kinetic energy to overcome the barrier set by the energy requirements of faster dissipative processes; in the present model, the dissipative processes are modeled by adding a viscous component to the interaction force during the early stages of the loading phase. This prevents nonphysical behaviors of the interaction law such as the unbounded penetration of an indenter in a drop test simulation [32].

Coupling these two steps, the piecewise-defined bit/rock interaction law summarized in Figure 5 is obtained. It comprises five different drilling modes, namely FF, dissipative forward contact (DFC), forward contact, backward contact (BC) and standstill (SS). Each corresponds to a specific motion of the bit/rock interface. Transition from one mode to the other operates at the occurrence of a condition associated with an arrow directed outward the current mode. Update of the history variables takes place at mode transitions; namely, the start of a new drilling cycle corresponds to switching to the DFC mode while peak penetration is reached when the interface law transitions to the BC mode.

Free flight corresponds to the absence of contact at the bit/rock interface; that is, the bit is off bottom; the force on the bit is therefore zero, $F_{R}=0$. At the closure of the gap at the bit/rock interface, $p=p_{u}$, the kinetic energy of the bit $\mathrm{KE}_{b}$ is evaluated and compared with the energy barrier $W_{R}$. If it is below the threshold, $\mathrm{KE}_{b} \leq W_{R}$, it is assumed that the bit has insufficient energy to achieve any significant penetration and the interaction law is set to the SS mode. In this mode, the bit is assumed to rest on a Kelvin-Voigt viscoelastic cell, with $F_{R}=\gamma K_{R}\left(p-p_{u}\right)+C_{R} \dot{p}$ and $\dot{p}=\mathrm{d} p / \mathrm{d} t$. If the bit has sufficient kinetic energy to overcome the barrier, $\mathrm{KE}_{b}>W_{R}$, the drilling mode is switched to DFC, the penetration while drilling is reset to zero, and a new drilling cycle is started. The interaction force is given by $F_{R}=K_{R} p+C_{R} \dot{p}$. The interaction law remains in this mode until either the energy dissipated by the viscous damping $W(\dot{p})=C_{R} \int_{0}^{t} \dot{p}^{2}(\bar{t}) \mathrm{d} \bar{t}$ becomes equal to the energy barrier, $W(\dot{p})=W_{R}$, in which case- the interaction law is then switched to the forward contact mode for which $F_{R}=K_{R} p_{\curlywedge}$ or the penetration velocity becomes negative, $\dot{p}=0_{2}$ in which case; the interaction law is switched to the BC mode. Upon entering the BC mode, the upper penetration is set to $p_{u}=p_{p}(\gamma-1) / \gamma$, on the basis of the peak penetration that is given by the penetration at the transition. The interaction force is given by $F_{R}=\gamma K_{R}\left(p-p_{u}\right)$. From that mode, two transitions are possible. Either the force on the bit vanishes at $p=p_{u}$ and the interaction law is switched to the FF mode or the velocity of the bit/rock interface changes its sign and the interaction law transitions to the DFC mode or the SS mode depending on the bit kinetic energy 


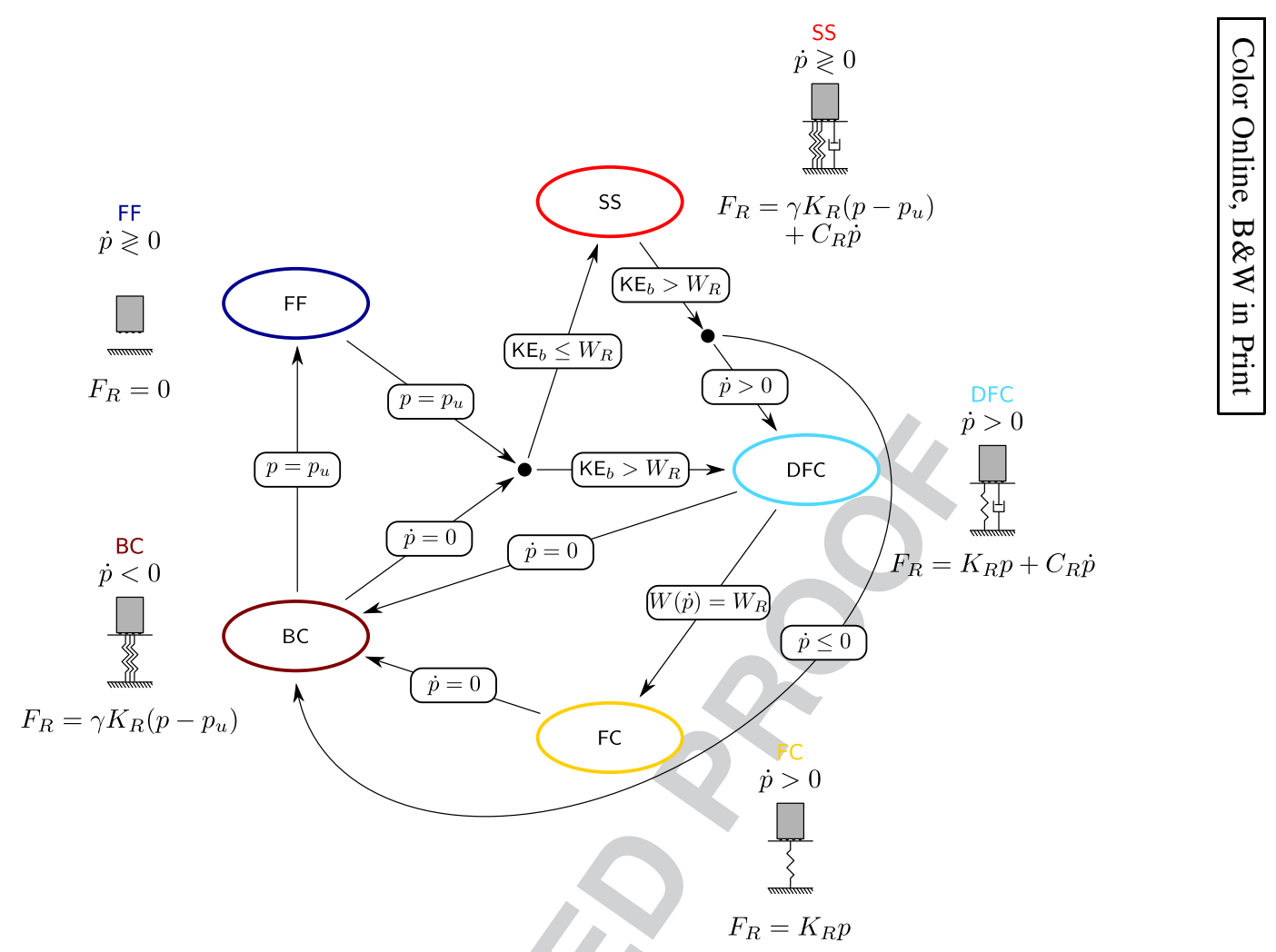

Figure 5. Piecewise definition of the bit/rock interaction, on the basis of the penetration while drilling $p$ (the drilling cycle index is dropped for legibility). The transition from one drilling mode to the other takes place when one of the conditions specified on the arrows outgoing the current mode is met. The overhead dot denotes time differentiation, $\dot{p}=\mathrm{d} p / \mathrm{d} t$. FF, free flight; BC, backward contact; FC, forward contact; SS, standstill; DFC, dissipative forward contact.

at the instant of the transition. The interaction law exits the SS mode when the bit kinetic energy becomes superior to the energy barrier, $\mathrm{KE}_{b}>W_{R}$. The next drilling mode then depends on the sign of the interface velocity.

The parameters of the bit/rock interaction law can be inferred from experiments, numerical simulations, and the model assumptions. Parameters $K_{R}$ and $\gamma$ can be obtained from drop test experiments (physical or numerical); for instance, the numerical simulations of Saksala [19, Erratum, Figure 10] who considers a 2-in drill bit with 10 hemispherical buttons indenting hard rock lead to $K_{R} \simeq 350 \mathrm{kN} / \mathrm{mm}$ and $\gamma \simeq 5$. Ideally, they should be evaluated from experiments that reproduce the drilling pattern, that is, on the basis of drop tests on a damaged surface and including the effect of the bit rotation between successive impacts. Parameters $W_{R}$ and $C_{R}$ must be identified by a calibration procedure, in respect of the scaling assumptions underlying their definition; that is, the dissipative processes (thus, the DFC phase following a percussive activation) are fast when compared with the duration of a typical drilling cycle and do not represent a significant part of the energy dissipated over a drilling cycle; additional details are given in Sections 2.4 and 4.2.

Inherent to the proposed formulation is the assumption that the effective penetration, which accounts for the partial removal of damaged rock by the flushing mechanism, is equal to the penetration of the bit buttons. This restriction could readily be relaxed, however, by assuming an empirical power law relation between the effective penetration, $p_{\mathrm{eff}}^{(n)}$, and the penetration at the end of a drilling cycle, $p^{(n)}\left(t_{n+1}^{-}\right)$

$$
p_{\mathrm{eff}}^{(n)}=\zeta_{1}\left(p^{(n)}\left(t_{n+1}^{-}\right)\right)^{\zeta_{2}}
$$


with $\zeta_{1}, \zeta_{2}>1$ to be determined experimentally. The increment of the internal variable $x_{R}^{(n)}$ would then be given by the effective penetration

$$
\Delta x_{R}\left(t_{n+1}\right)=p_{\mathrm{eff}}^{(n)} .
$$

Accordingly, the internal variable $x_{R}^{(n)}$ would represent the position of the rock surface and the transition BC $\rightarrow$ FF would be the only possible transition out of the BC drilling mode; the continuity of the interaction force is not enforced during this transition.

\subsection{Model intrinsic scales}

By nature, the percussive drilling process spans multiple scales, with small-scale processes driving large-scale processes. Further examination of the governing equations reveals that, despite its simplified nature, the model also spans multiple reference scales for both the time and length-units. In particular, from the dynamics of the drilling (sub)processes and the model parameters (Table I; parameters $x_{R}$ and $\beta_{R}$ generically refer to the history variables and the drilling mode), the following scales can be identified.

Two timescales can be associated with the wave propagation in the bit and the piston; they are given by the wave travel times in each body

$$
T_{1}=\frac{L_{p}}{c_{0}}, \quad T_{2}=\frac{L_{b}}{c_{0}},
$$

with $c_{0}=\sqrt{E / \rho}$ the wave propagation speed in the material. Recalling the scaling of the pressure law, a third timescale, approximating the piston FF time, is given by

$$
T_{3}=\sqrt{\frac{M_{p} D_{1}}{F_{0}}},
$$

where $M_{p}=\rho A L_{p}$ denotes the piston mass. On the basis of rigid body motion considerations, the duration of a drilling cycle can be shown to scale with

$$
T_{4}=\sqrt{\frac{M_{b}}{K_{R}}},
$$

where $M_{b}=\rho A L_{b}$ refers to the bit mass. A fifth timescale associated with the energy barrier can also be identified. Assuming the bit velocity to be constant and equal to $V_{P}=\sqrt{F_{0} D_{1} / M_{p}}$, the

Table I. Parameters entering the governing equations and their generic units (M: mass, L: length, and T: time).

\begin{tabular}{lllllll}
\hline Variables & & & & & & \\
\hline Dependent & $u_{p}$ & $\mathrm{~L}$ & $u_{b}$ & $\mathrm{~L}$ & & \\
Independent & $t$ & $\mathrm{~T}$ & $X_{p}$ & $\mathrm{~L}$ & $X_{b}$ & $\mathrm{~L}$ \\
Parameters & & & & & & \\
Material & $E$ & $\mathrm{ML}^{-1} \mathrm{~T}^{-2}$ & $\rho$ & $\mathrm{ML}^{-3}$ & & \\
Geometry & $L_{p}$ & $\mathrm{~L}$ & $L_{b}$ & $\mathrm{~L}$ & $A$ & $\mathrm{~L}^{2}$ \\
Static loads & $G$ & $\mathrm{LT}^{-2}$ & $F_{S}$ & $\mathrm{MLT}^{-2}$ & & \\
Pressure law & $D_{1}$ & $\mathrm{~L}$ & $F_{0}$ & $\mathrm{MLT}^{-2}$ & $\alpha$ & 1 \\
Bit/rock interaction & $K_{R}$ & $\mathrm{MT}^{-2}$ & $C_{R}$ & $\mathrm{MT}^{-1}$ & $W_{R}$ & $\mathrm{ML}^{2} \mathrm{~T}^{-2}$ \\
& $\gamma$ & 1 & $\beta_{R}$ & 1 & $x_{R}$ & $\mathrm{~L}$ \\
Contact & $g_{0}$ & $\mathrm{~L}$ & & & & \\
\hline
\end{tabular}


integral condition involved in the definition of defining-the energy barrier can be solved for its upper boundary, yielding

$$
T_{5}=\frac{W_{R}}{F_{0} D_{1}} \frac{M_{p}}{C_{R}}
$$

This approximation only holds for the drilling cycle ensuing a percussive activation; indeed, the lower bit velocity in subsequent drilling cycles induces a larger duration of the DFC phase. It can be used to define the values of the energy barrier parameters $C_{R}$ and $W_{R}$. In particular, the constraints

$$
T_{5} / T_{4} \ll 1, \quad W_{R} / F_{0} D_{1} \ll 1
$$

must be enforced to reflect the faster and lower energy nature of the processes underlying the existence of the energy barrier. Typical orders of magnitude for the timescales read (in milliseconds)

$$
T_{1}=O\left(10^{-2}\right), \quad T_{2}=O\left(10^{-2}\right), \quad T_{3}=O(10), \quad T_{4}=O\left(10^{-1}\right), \quad T_{5}=O\left(10^{-4}\right) .
$$

These clearly emphasize the multiscale nature of the model, as five orders of magnitude are spanned by the different timescales for a typical configuration.

Parallel to the timescales, multiple length scales can be identified. We list two of them, namely the piston length and the second as a typical penetration following a percussive activation

$$
L_{1}=L_{p}, \quad L_{2}=\sqrt{\frac{F_{0} D_{1}}{K_{R}}} .
$$

They have magnitudes (in millimeters)

$$
L_{1}=O(100), \quad L_{2}=O(0.1) .
$$

\subsection{Model position}

The dimensionless group $\eta=K_{R} L_{b} / E A$, equal to the timescale ratio $\eta=T_{2}^{2} / T_{4}^{2}$, plays an essential role on the nature of the bit behavior before the rock. Indeed, this parameter indicates whether the bit behaves as a rigid body $(\eta \ll 1)$ or an elastic one $(\eta \gg 1)$ when it interacts with the rock [5, 32].

For DTH percussive drilling, this parameter typically ranges below $1, \eta<1$, and the bit mainly behaves as a rigid body when it penetrates the rock. On the contrary, its interaction with the piston during percussive activation is ruled by wave propagation, given their similar impedances and lengths. This justifies the need for an elastic representation of the piston and the bit.

For TH percussive drilling, $\eta>1$, typically, given that $L_{b}$ includes the length of the drill steel. An elastic representation of the piston and the bit is thus required to represent both the bit/rock interaction and the percussive activation.

\section{COMPUTATIONAL MODEL}

The partial differential equations governing the dynamics of the proposed model are infinite dimensional. For computational purposes, these have to be approximated by a finite dimensional problem. The numerical results presented in Section 4 are based on a semidiscrete approximation using linear finite elements in space [33] and an event-driven time integration procedure [16] that takes care of the piecewise linear nature of model equations; the contact interaction at the piston/bit interface is handled via the penalty method [34]. 


\subsection{Semidiscrete model}

Assuming the displacement field to be of the separated form

$$
u_{i}\left(X_{i}, t\right)=\mathcal{Z}_{i}\left(X_{i}\right) \mathcal{U}_{i}(t), \quad i \in\{p ; b\},
$$

the application of the finite element method $[20,33]$ to the scalar wave equation ruling the piston and the bit motions becomes a mere exercise. Accounting for the piecewise definition of the boundary conditions, the semidiscrete approximation can be written in the following form

$$
\left(\begin{array}{cc}
\mathbf{0} & \mathbf{M} \\
\mathbf{I} & \mathbf{0}
\end{array}\right)\left(\begin{array}{c}
\dot{\mathcal{U}} \\
\dot{\mathcal{V}}
\end{array}\right)+\left(\begin{array}{cc}
\mathbf{K}+\mathbf{K}_{C}+\mathbf{K}_{R} & \mathbf{C}_{R} \\
\mathbf{0} & -\mathbf{I}
\end{array}\right)\left(\begin{array}{l}
\mathcal{U} \\
\mathcal{V}
\end{array}\right)=\left(\begin{array}{c}
\mathbf{f}+\alpha \mathbf{f}_{P}+\mathbf{f}_{C}+\mathbf{f}_{R} \\
\mathbf{0}
\end{array}\right)
$$

where, as usual, the overhead dot denotes time differentiation, $\dot{x}(t)=\mathrm{d} x / \mathrm{d} t$. Vectors $\mathcal{U}, \mathcal{V} \in \mathbb{R}^{n}$ contain the displacements and the velocities of the $n$ degrees of freedom of the semidiscrete model. Structural matrices $\mathbf{K}, \mathbf{M} \in \mathbb{R}^{n \times n}$ follow from the semidiscretization procedure; they depend on the interpolation order of the shape functions as well as on the mesh used. Given the nonsmooth nature of the model, we use linear elements; a $_{2}$ uniform mesh is used. Accordingly, the stiffness and mass matrices are symmetric and, respectively, positive semidefinite, $\mathbf{K} \geq 0$, and positive definite, $\mathbf{M}>0$, as they result from the assembly of the following element matrices $\overline{\bar{\Delta}}$

$$
\mathbf{K}_{e}=\frac{E A}{L_{e}}\left(\begin{array}{rr}
1 & -1 \\
-1 & 1
\end{array}\right), \quad \mathbf{M}_{e}=\frac{\rho A L_{e}}{6}\left(\begin{array}{ll}
2 & 1 \\
1 & 2
\end{array}\right),
$$

where the element length, denoted by $L_{e}$, is constant over each body; the consistent mass matrix is used. As to $\mathbf{f}$, the vector of external loads, it can be expressed by a composition of nodal accelerations projected in the mass matrix

$$
\mathbf{f}=\mathbf{M}\left(G \mathbf{1}_{p}+\left(G+\frac{F_{S}}{M_{b}}\right) \mathbf{1}_{b}\right) .
$$

Vectors $\mathbf{1}_{i} \in \mathbb{R}^{n}, i \in\{p, b\}$, have unit entries at rows associated with the degrees of freedom of body $i$ and zero entries at other rows. The subscripted quantities are piecewise defined and vary with the configuration of the model; these are related to the definition of (i) the contact force at the piston/bit interface (subscript $C$ ), (ii) the pressure force on the piston (subscript $P$ ), and (iii) the bit/rock interaction force at the bit bottom (subscript $R$ ).

\subsection{Piecewise-defined quantities}

The update of piecewise-defined quantities takes place each time a transition occurs in the model. Each transition is represented by an algebraic event function that goes through zero at the instant the transition occurs.

The handling by the penalty method of the contact interaction at the piston/bit interface is based on the definition of the gap function

$$
g=g_{0}+\mathbf{w}_{C}^{T} \mathcal{U}
$$

that quantifies the opening between the piston bottom end and the bit top end. Vector $\mathbf{w}_{C} \in \mathbb{R}^{n}$ is a signed localization vector that identifies the degrees of freedom associated with the contact interface; $g_{0}$ denotes the initial gap. Following this definition, the complementarity condition expressing the unilateral nature of the contact interaction can be relaxed by expressing the contact force as a function of the interpenetration of the contacting interfaces

$$
\lambda_{C}=-K_{C} \mathbf{w}_{C}[g]_{-},
$$

with the Macaulay brackets defined as $[g]_{-}=\min \{0 ; g\}$; the proportionality constant $K_{C}$ is known as the contact stiffness. Thus, the magnitude of the contact force is zero when the interface is open 
(positive gap value, $g>0$ ) and positive when the contacting bodies interpenetrate (negative gap value, $g \leq 0$ ). Changes of contact status are thus related to the gap function going through zero, which yields the first event function

$$
q_{1}=g
$$

From the definition of the contact force $\lambda_{C}$, we readily see that

$$
\begin{array}{c|cc} 
& q_{1}<0 & q_{1}>0 \\
\hline \mathbf{K}_{C} & K_{C} \mathbf{w}_{C} \mathbf{w}_{C}^{T} & \mathbf{0} \\
\mathbf{f}_{C} & -K_{C} \mathbf{w}_{C} g_{0} & \mathbf{0}
\end{array}
$$

Switches from one column to the other occur whenever $q_{1}=0$. Grazing situations, corresponding to $\dot{q}_{1}=0$, shall not be encountered by design of the event-driven procedure that is used and are thus not a concern.

The pressure force driving the piston is defined on the basis of the average motion of the piston relative to that of the bit. It is upwards after the occurrence of percussive activation and so until the relative displacement becomes of amplitude larger than $D_{1}$. It is directed downwards otherwise. This naturally calls for the following two event functions that trigger the switches of $\alpha$, the force direction:

$$
\begin{gathered}
q_{2}=\mathcal{U}_{r}, \\
q_{3}=\mathcal{U}_{r}+D_{1},
\end{gathered}
$$

where the average relative motion $\mathcal{U}_{r}$ is defined as

$$
\mathcal{U}_{r}=\left\langle\mathcal{U}_{p}\right\rangle_{X}-\left\langle\mathcal{U}_{b}\right\rangle_{X}-g_{0}
$$

with the piston and bit average displacements obtained by projection in the mass matrix

$$
\left\langle\mathcal{U}_{p}\right\rangle_{X}=\frac{\mathbf{1}_{p}^{T} \mathbf{M} \mathcal{U}}{\mathbf{1}_{p}^{T} \mathbf{M} 1_{p}}, \quad\left\langle\mathcal{U}_{b}\right\rangle_{X}=\frac{\mathbf{1}_{b}^{T} \mathbf{M} \mathcal{U}}{\mathbf{1}_{b}^{T} \mathbf{M} \mathbf{1}_{b}} .
$$

This projection is equivalent to the calculation of integrals (10) using the linearly interpolated field, given the use of linear elements and that of the consistent mass matrix. The cycle of switches is given as follows. Assuming $\alpha=1$ initially, that is, an initially downward pressure force, $\alpha=-1$ is set when $q_{2}$ vanishes. It is then reset to $\alpha=1$ when $q_{3}$ vanishes. We assume that event functions $q_{2}$ and $q_{3}$ are mutually exclusive, which is a slight deviation to the definition (11) that reinforces the character of piston reciprocating motion; only one is to be tracked at a given time, depending on the sign of $\alpha$. The distributed pressure force is also obtained by the projection of the uniform acceleration field $F_{0} / M_{p}$ in the mass matrix

$$
\mathbf{f}_{P}=\frac{F_{0}}{M_{p}} \mathbf{M} \mathbf{1}_{p}
$$

Event functions and piecewise-defined quantities relative to the bit/rock interaction law are immediately identified from Figure 5 and the definition of the penetration while drilling

$$
\mathcal{P}=\mathbf{w}_{R}^{T} \mathcal{U}-\mathcal{X}_{R}
$$

Vector $\mathbf{w}_{R} \in \mathbb{R}^{n}$ identifies the degree of freedom located at the bit/rock interface; history variable $\mathcal{X}_{R}$ is equivalent to internal variable $x_{R}^{(n)}$ and represents the shifted position of the rock surface at the lower location along the drilling cycle. The penetration while drilling is reset at the start of a 
new drilling cycle (transition to DFC mode), to zero if coming from the FF mode or to a residual penetration if coming from either the BC or the SS mode. The upper penetration $\mathcal{P}_{u}=\mathcal{P}_{p}(\gamma-1) / \gamma$ is set at the transition to the $\mathrm{BC}$ mode and serves the definition of the upper bit position

$$
\mathcal{X}_{b, u}=\mathcal{P}_{u}+\mathcal{X}_{R}
$$

Indeed, following these definitions, the interaction force can generically be stated as

$$
\lambda_{R}=-\mathbf{w}_{R}\left(\mathcal{K}_{R}\left(\mathcal{P}-\mathcal{P}_{R}\right)+\mathcal{C}_{R} \dot{\mathcal{P}}\right),
$$

yielding the structure of the piecewise-defined quantities

$$
\mathbf{K}_{R}=\mathcal{K}_{R} \mathbf{w}_{R} \mathbf{w}_{R}^{T}, \quad \mathbf{C}_{R}=\mathcal{C}_{R} \mathbf{w}_{R} \mathbf{w}_{R}^{T}, \quad \mathbf{f}_{R}=\mathcal{K}_{R} \mathcal{P}_{R} \mathbf{w}_{R} .
$$

The parameters $\mathcal{K}_{R}, \mathcal{C}_{R}$, and $\mathcal{P}_{R}$, dependent on the drilling mode, and the event functions then read

\begin{tabular}{l|ccc|l} 
& $\mathcal{K}_{R}$ & $\mathcal{C}_{R}$ & $\mathcal{P}_{R}$ & \multicolumn{1}{|c}{$q_{i}$} \\
\hline FF & 0 & 0 & 0 & $q_{4}=\mathcal{P}-\mathcal{P}_{u}$ \\
DFC & $K_{R}$ & $C_{R}$ & $\mathcal{X}_{R}$ & $q_{5}=W(\dot{\mathcal{P}})-W_{R} q_{6}=\dot{\mathcal{P}}$ \\
FC & $K_{R}$ & 0 & $\mathcal{X}_{R}$ & $q_{7}=\dot{\mathcal{P}}$ \\
BC & $\gamma K_{R}$ & 0 & $\mathcal{X}_{b, u}$ & $q_{8}=\dot{\mathcal{P}}$ \\
SS & $\gamma K_{R}$ & 0 & $\mathcal{X}_{b, u}$ & $q_{10}=\mathrm{KE}_{b}-W_{R}=\mathcal{P}-\mathcal{P}_{u}$
\end{tabular}

where, for completeness, the kinetic energy of the bit is given by

$$
\mathrm{KE}_{b}=\frac{1}{2}\left(\mathbf{I}_{b} \mathcal{V}\right)^{T} \mathbf{M}\left(\mathbf{I}_{b} \mathcal{V}\right)_{\triangle}
$$

with $\mathbf{I}_{b}$ as the restriction of the identity matrix to the bit degrees of freedom and

$$
W(\dot{\mathcal{P}})=C_{R} \int_{0}^{t}(\dot{\mathcal{P}}(t))^{2} \mathrm{~d} t
$$

the work performed by the viscous component of the bit/rock interaction force. Again, only the event functions related to the current drilling mode are to be considered for the update of the piecewisedefined quantities; for example, only event function $q_{4}$ is to be considered while in the FF mode.

\subsection{Event-driven integration}

The piecewise formulation of the computational model naturally calls for an event-driven integration procedure, that is, an integration procedure that accurately locates the occurrence of events while performing the time integration of the governing equations $[35,36]$. Such a procedure requires the combination of an integration scheme and a root-solving module.

The results presented in Section 4 are based on the $\mathrm{DE}^{3}$ integration scheme, presented in [37], and on an adaptation of the root-solving module detailed in [16]. Specific to these components are the unconditional stability of the one-step integration scheme, as well as the use of Hermite interpolation to approximate the event functions between time steps and the driving of event-localization past the event occurrence by a relaxation procedure. The latter feature prevents the occurrence of grazing behaviors. The integral of (53) is evaluated consistently with the integration scheme, on the basis of energy balance results. All algorithmic details can be found in [32].

\subsection{Taming the spurious oscillations}

Of notorious knowledge is the occurrence of Gibbs (spurious) oscillations when numerically simulating nonsmooth problems. This is particularly true for impact problems in 1D elasticity, given the 
discontinuous nature of the strain and velocity fields following the collisions [21]. These nonphysical oscillations must be dealt with to avoid perturbations of the switch model defining the bit/rock interaction law.

Finite difference upwind schemes have become one of the state-of-the-art numerical techniques to simulate wave propagation, for they sensibly reduce the spurious oscillations around wave fronts and enable the development of high-order schemes for smooth problems. From the modified equations relative to the first-order explicit scheme developed by Banks and Henshaw [38], it appears that a parallel can be made between stiffness-proportional damping and the damping effect associated with upwinding. Numerical experiments [32] have confirmed this similarity and shown that nonoscillatory impact responses in 1D elastic bars can be recovered with the semidiscrete approach we follow, provided the amount of stiffness-proportional damping is adequately adjusted.

Accordingly, we introduce viscous damping proportional to the stiffness matrix in the semidiscrete equations of motion. They now read

$$
\left(\begin{array}{cc}
\mathbf{0} & \mathbf{M} \\
\mathbf{I} & \mathbf{0}
\end{array}\right)\left(\begin{array}{l}
\dot{\mathcal{U}} \\
\dot{\mathcal{V}}
\end{array}\right)+\left(\begin{array}{cc}
\mathbf{K}+\mathbf{K}_{C}+\mathbf{K}_{R} \mathbf{C}+\mathbf{C}_{R} \\
\mathbf{0} & -\mathbf{I}
\end{array}\right)\left(\begin{array}{l}
\mathcal{U} \\
\mathcal{V}
\end{array}\right)=\left(\begin{array}{c}
\mathbf{f}+\alpha \mathbf{f}_{P}+\mathbf{f}_{C}+\mathbf{f}_{R} \\
\mathbf{0}
\end{array}\right)
$$

with

$$
\mathbf{C}=2 \frac{\zeta_{K}}{\omega_{\mathrm{cr}}} \mathbf{K}
$$

and $\zeta_{K}$ the damping ratio associated with the eigenmode of highest frequency $\omega_{\mathrm{cr}}$.

\section{NUMERICAL RESULTS}

\subsection{Reference configuration and numerical settings}

The numerical results presented in the ensuing analysis are based on a set of reference values representative of a $3 \frac{1}{4}$-in DTH percussive system drilling hard rock. These are listed in Table II, as well as the reference scales and the numerical simulation parameters.

For all simulations, the spatial mesh is taken uniform with $e_{p}=50$ elements on the piston and $e_{b}=\left\lceil e_{p} L_{b} / L_{b}\right\rceil=58$ on the bit, to ensure similar element sizes on both bodies. Following a convergence analysis [32], the contact stiffness has been set to $10 \%$ of the largest entry of the stiffness

Table II. Reference configuration; the units are mm, ms, N, MPa, and

\begin{tabular}{|c|c|c|c|}
\hline \multicolumn{4}{|c|}{ Model parameters } \\
\hline $\begin{array}{l}\text { Material } \\
\text { Geometry } \\
\text { Static loads } \\
\text { Pressure law } \\
\text { B/R interaction law }\end{array}$ & $\begin{aligned} E & =210 \cdot 10^{3} \\
L_{p} & =175 \\
G & =10^{-2} \\
F_{0} & =3000 \\
K_{R} & =750 \cdot 10^{3} \\
C_{R} & =10^{4}\end{aligned}$ & $\begin{aligned} \rho & =7850 \cdot 10^{-6} \\
L_{b} & =201.25 \\
F_{S} & =2500 \\
D_{1} & =40 \\
\gamma & =5 \\
W_{R} & =50\end{aligned}$ & $A=5500$ \\
\hline \multicolumn{4}{|l|}{ Reference scales } \\
\hline $\begin{array}{l}\text { Time } \\
\text { Length }\end{array}$ & $\begin{aligned} T_{1} & =3.38 \cdot 10^{-2} \\
T_{4} & =0.108 \\
L_{1} & =175\end{aligned}$ & $\begin{aligned} T_{2} & =3.87 \cdot 10^{-2} \\
T_{5} & =3.15 \cdot 10^{-4} \\
L_{2} & =0.40\end{aligned}$ & $T_{3}=10.0$ \\
\hline \multicolumn{4}{|l|}{ Numerical parameters } \\
\hline $\begin{array}{l}\text { Degrees of freedom } \\
\text { Contact } \\
\text { Structural damping } \\
\text { Root-solving }\end{array}$ & $\begin{aligned} e_{p} & =50 \\
K_{C} & =10^{9} \\
\zeta_{K} & =0.5 \\
\text { qTol } & =10^{-6}\end{aligned}$ & $\begin{aligned} e_{b} & =58 \\
\omega_{\mathrm{cr}} & =5163.7 \\
\mathrm{tTol} & =10^{-4}\end{aligned}$ & $\operatorname{degTol}=10^{-12}$ \\
\hline
\end{tabular}
rad/ms, $\mathrm{N} / \mathrm{mmm}_{2}$

$\mathrm{B} / \mathrm{R}=$ bit/rock. 
matrix and the time step has been set to five times the CFL number associated with the smallest element size. The time integration scheme is used in its spectrally annihilating configuration, thereby providing a fast damping for high-frequency spurious oscillations, which are further attenuated via proportional damping so that a level of $50 \%$ of the critical damping associated with the highestfrequency eigenmode is achieved. The root-solving tolerances, qTol, tTol, and degTol, have also been chosen according to a sensitivity analysis [16].

All forward simulations are initialized in a generalized FF configuration. By this designation, it is meant that all contact interfaces are initially open, that the piston has downward uniform velocity, and that the bit is uniformly at rest above the rock (no initial deformation). Thus, the initial gap at the piston/bit interface is given a positive value $g_{0}>0$ and the bit/rock interaction law is initialized in the FF mode with a positive bit/rock opening $\mathcal{P}_{u}>0$. Also, in this initial setting, it is assumed that the pressure force acts in the downward direction, viz, $\alpha=1$. The definition of these conditions is such that a percussive activation is expected in the early simulation times.

\subsection{Influence of the energy barrier parameters}

The definition of the energy barrier introduced by the authors to generalize the bilinear bit/rock interaction law to successive drilling cycles is based on two parameters, $C_{R}$ and $W_{R}$. Given the assumptions underlying their definition, these parameters must be defined such that (30) holds. These conditions leave some freedom as to their definition, however.

To illustrate the influence these parameters play on the response of the bit penetration, we simulate the drop test of the bit on the rock; see the setup of the model problem in Figure 6(a). Such simulations can be performed on the basis of the proposed model, after removal of the components relative to the piston. FF initial conditions are retained for the simulation, with the bit initial kinetic energy set to the energy injected by the pressure force over a pressure cycle, $\mathrm{KE}_{b}=F_{0} D_{1}$; that is, $\left(\left\langle\mathcal{V}_{b}\right\rangle_{X}, \mathcal{P}_{u}\right)=\left(5.25 \mathrm{~mm} / \mathrm{ms}, 10^{-2} \mathrm{~mm}\right)$, with the average bit velocity

$$
\left\langle\mathcal{V}_{b}\right\rangle_{X}=\frac{\mathbf{1}_{b}^{T} \mathbf{M} \mathcal{V}}{\mathbf{1}_{b}^{T} \mathbf{M} \mathbf{1}_{b}}
$$

The bit is assumed initially stress free.

Figure 6(b) shows the influence of the energy barrier parameters on the time evolution of the bit average displacement that consists of a succession of drilling cycles and FF phases prior to convergence to SS. Several effects are visible. First, the energy barrier $W_{R}$ influences the number of drilling cycles the bit completes prior to entering the SS mode; the larger the $W_{R}$, the smaller this number. Second, the larger the energy barrier $W_{R}$, the more energy is dissipated per drilling cycle. Accordingly, the penetration per drilling cycle decreases with $W_{R}$. Third, the smaller the magnitude of the energy barrier $W_{R}$, the less influence the damping coefficient $C_{R}$ plays on the bit displacement.

As witnessed by the results of Figure 6(c), it is the magnitude of the energy barrier $W_{R}$ that plays a major role on the achieved bit penetration. A large value can reduce it by tens of per-cent. Given these results, a calibration procedure based on the response of a drop test restrict the range of values of $C_{R}$ and $W_{R_{\Sigma}}$ meeting the conditions (30).

\subsection{Stationary response at reference configuration}

Simulations show that an attractive period-1 solution exists for the reference configuration; it can be reached from generalized FF initial conditions after a sufficiently long time integration of the model equations. The results presented hereafter are based on the initial settings $\left(\left\langle\mathcal{V}_{p}\right\rangle_{X}, g_{0}, \mathcal{P}_{u}\right)=$ $\left(10 \mathrm{~mm} / \mathrm{ms}, 10^{-2} \mathrm{~mm}, 10^{-2} \mathrm{~mm}\right)$, with

$$
\left\langle\mathcal{V}_{p}\right\rangle_{X}=\frac{\mathbf{1}_{p}^{T} \mathbf{M} \mathcal{V}}{\mathbf{1}_{p}^{T} \mathbf{M} \mathbf{1}_{p}}
$$

Several projections of the periodic solution are proposed, so that it can be observed from several standpoints. The initial time has been set to correspond to the initiation of percussive activation. 


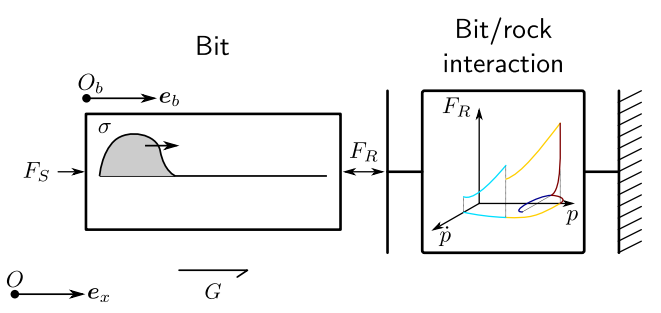

(a)

(b)
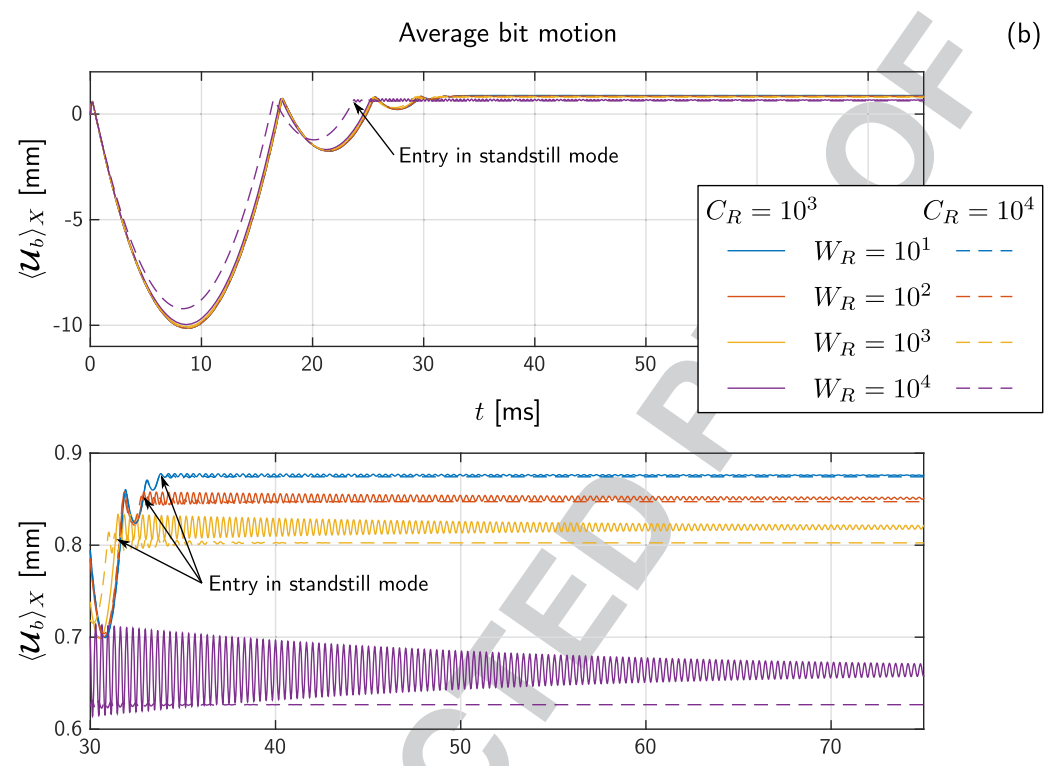

End penetration

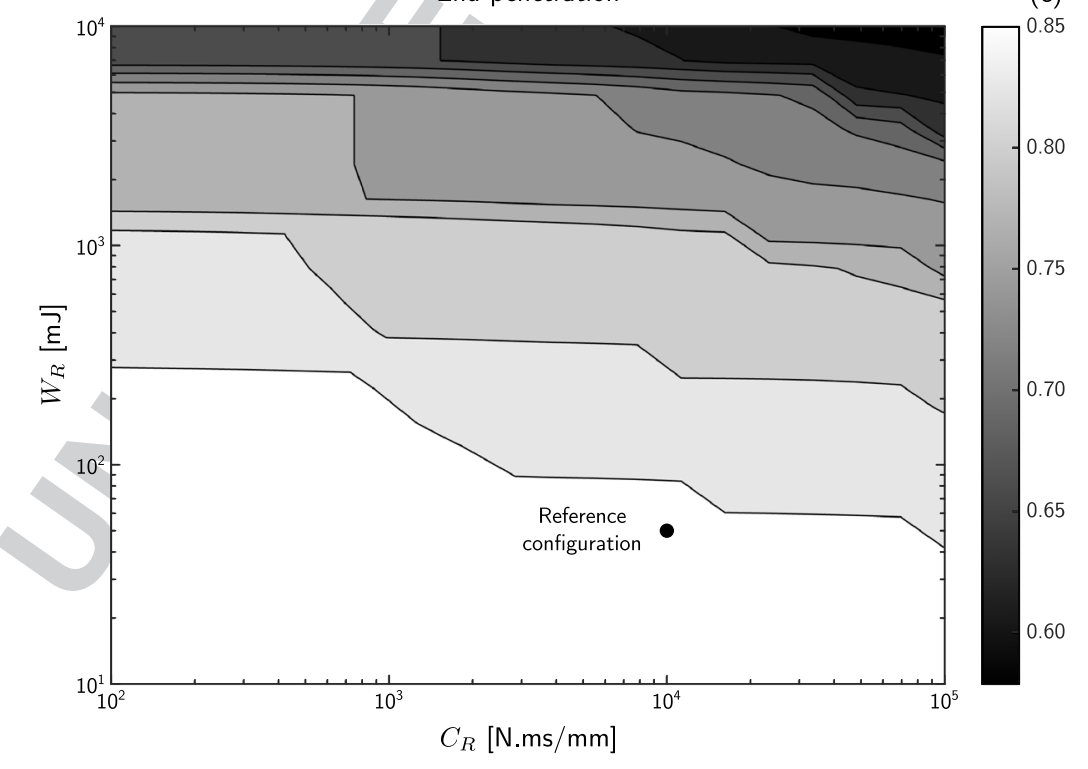

Figure 6. (a) Drop test configuration; (b) time evolution of the average bit motion as a function of the energy barrier parameters $C_{R}$ and $W_{R}$; and (c) influence of the energy barrier parameters $C_{R}$ and $W_{R}$ on the bit end penetration. 
Figure 7 shows the time series of the average bit motion and of the bit/rock interaction force over one period of the limit cycle. Also, the phase portrait associated with the bit average motion and the force/displacement response of the bit/rock interaction law are shown. Colors are used to represent the current drilling mode of the system, on top of the represented trajectories.

Bit motion can be decomposed in two main phases, as was already observed for the response of a drifting oscillator model of the drilling process [31]. The first one follows the percussive activation.
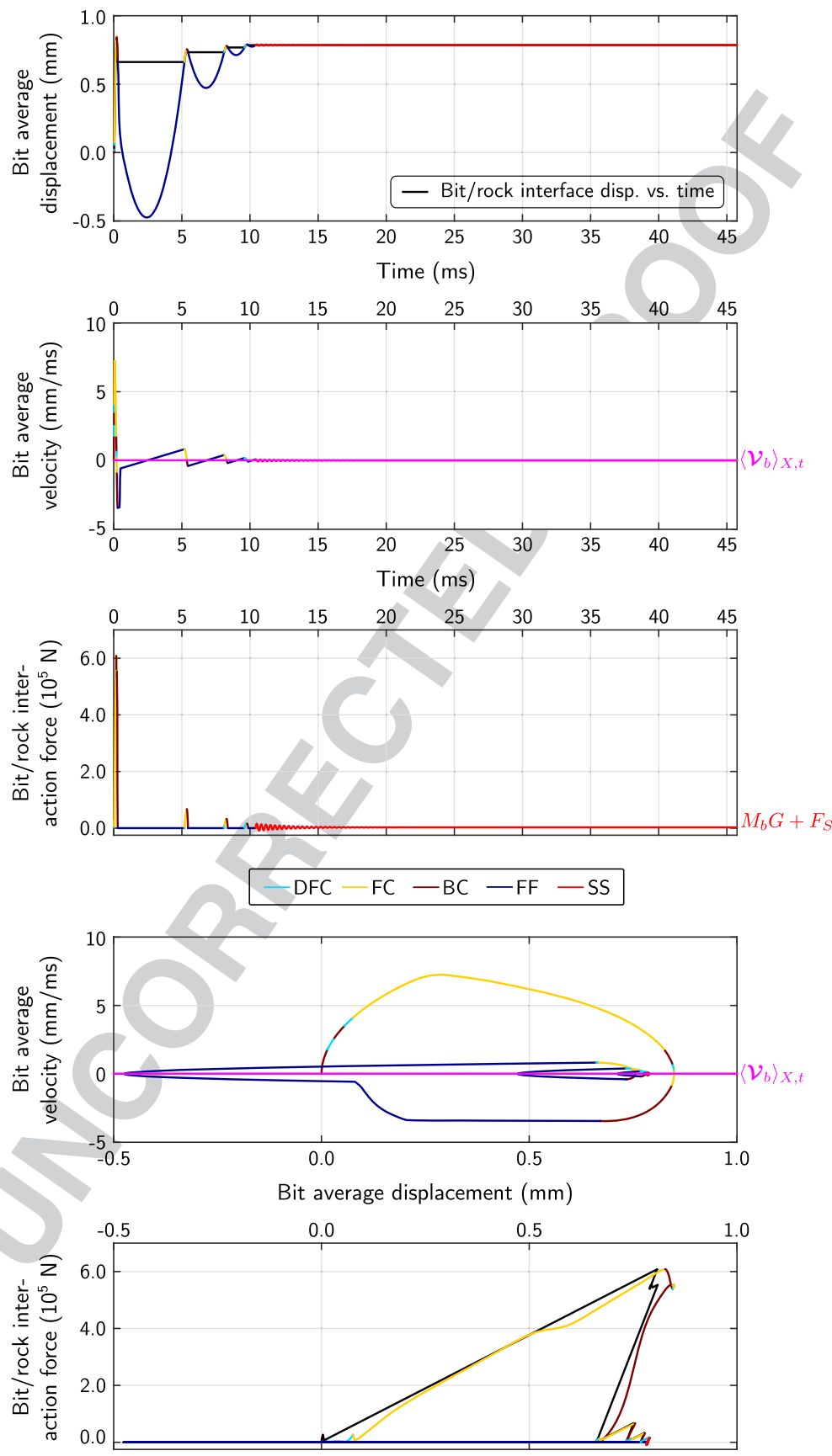

Figure 7. Period-1 solution corresponding to the reference configuration. Time series of the bit average motion and of the interaction force, as well as projections of the trajectory into the phase plane and of the bit/rock interaction response in the force/displacement plane. Color codes denote the current drilling mode. DFC, dissipative forward contact; FC, forward contact; BC, backward contact; FF, free flight; SS, standstill. 
It consists of a sequence of drilling cycles separated by FF phases of decreasing amplitudes; the pattern is similar to the one observed in drop test simulations. As can be noted, the peak penetration reached after the percussive activation is of the order of the millimeter. This is one order below the typical diameter of bit buttons. The saturation of the penetration following from the contact of the bit face with the rock is thus not to be expected. The second phase is that of SS; during which, the bit is in contact with the rock and converges to rest. Upon reaching equilibrium, static prevails over the bit dynamics and the bit/rock interaction thus converges to the apparent weight on the-bit, $F_{R} \rightarrow M G+F_{S} \simeq 2587 \mathrm{~N}$. This dichotomy of the bit motion is due to the activation period being larger than the convergence time to SS; indeed, the activation period (frequency) is about $45.5 \mathrm{~ms}$ ( $\equiv 22 \mathrm{~Hz}$ ) while convergence to standstill is achieved in about $10 \mathrm{~ms}$. Thus, in essence, all the energy brought to the bit by the percussive activation is consumed by the penetration process long before the piston returns for the next activation.

At the reference configuration, we have $\eta=O\left(10^{-1}\right)$; timescale separation $(\eta \ll 1)$ is thus not verified sensu stricto, and the response of the bit interacting with the rock is dominated by rigid body dynamics with some influence of elastic wave propagation. The effect of elastic waves propagating in the system can be observed multiple times in the results. First, a phase lag is observed between the average bit motion and the transitions between the drilling modes that are associated with the motion of the degree of freedom at the bit/rock interface; for instance, BC motion is observed while the bit experiences a positive average velocity. Second, the impact at the piston/bit interface leads to a continuous variation of their velocity, which is opposed to the discontinuous evolution enforced when modeling the collision of rigid bodies. Third, fluctuations around the bilinear backbone of the force/displacement are observed; the bilinear characteristic is recovered when representing the interaction force as a function of the displacement at the bit/rock interface.

The multiscale nature of the model is also clearly revealed by the time series. DFC phases are indeed observed to have a shorter duration than drilling cycles, which themselves are shorter than the period of percussive activation. This translates the timescale order $T_{3}>T_{4}>T_{5}$ observed in Table II.

An interesting behavioral feature is revealed by the phase portrait of the bit average motion and further illustrated in the time series of Figure 8 that shows the model response in the post-activation time range. It is the occurrence of two successive contact phases at the piston/bit interface. Following the percussive activation, the bit penetrates into the rock medium and then bounces off upon completing the drilling cycle. As, in this case, the normal gap velocity at the piston/bit interface is negative, $\dot{g}<0$, a second impact takes place. During that second contact phase, the bit acts as the collision driver and returns momentum to the piston, thereby accelerating it. This phenomenon, which is known to occur in physical devices [17, 39], is in fact desired, as it enables the increase of the percussive activation frequency and, in fine, the overall drilling performance. It also shows the need to include the piston dynamics in the model, for capturing such double-impact percussive activations with a drifting oscillator model that considers the sole bit motion is not possible. The double impact is the reason the bit converges to SS faster than it does in the drop test simulation.

In addition to the time series, Figure 8 shows the relative average motion of the system. The overall response is similar to the approximation that was derived in the definition of the pressure law, on the basis of a fixed bit; see Section 3.2. The change of relative acceleration coincides with the relative displacement crossing the pressure switch point at $\mathcal{U}_{r}=-D_{1}$, and the two impacts are identified as the zones of abrupt velocity variations near zero relative displacement in the relative phase portrait (segments $A B$ and $C D$ ). Positive relative displacements mainly result from the compliance of both bodies and, to a lesser extent, from the use of the penalty method to enforce the contact interaction; indeed, the interpenetration at the piston/bit interface is of the order of $g=10^{-3} \mathrm{~mm}$, as can be estimated from the value of the contact force and that of the contact stiffness.

\subsection{Influence of model parameters}

To further explore the predictions of the computational model, the influence of the model parameters on the average rate of penetration has been assessed, around the reference configuration. Four parameters have been considered: the bit/rock interaction parameters $K_{R}$ and $\gamma$, the feed force $F_{S}$, 

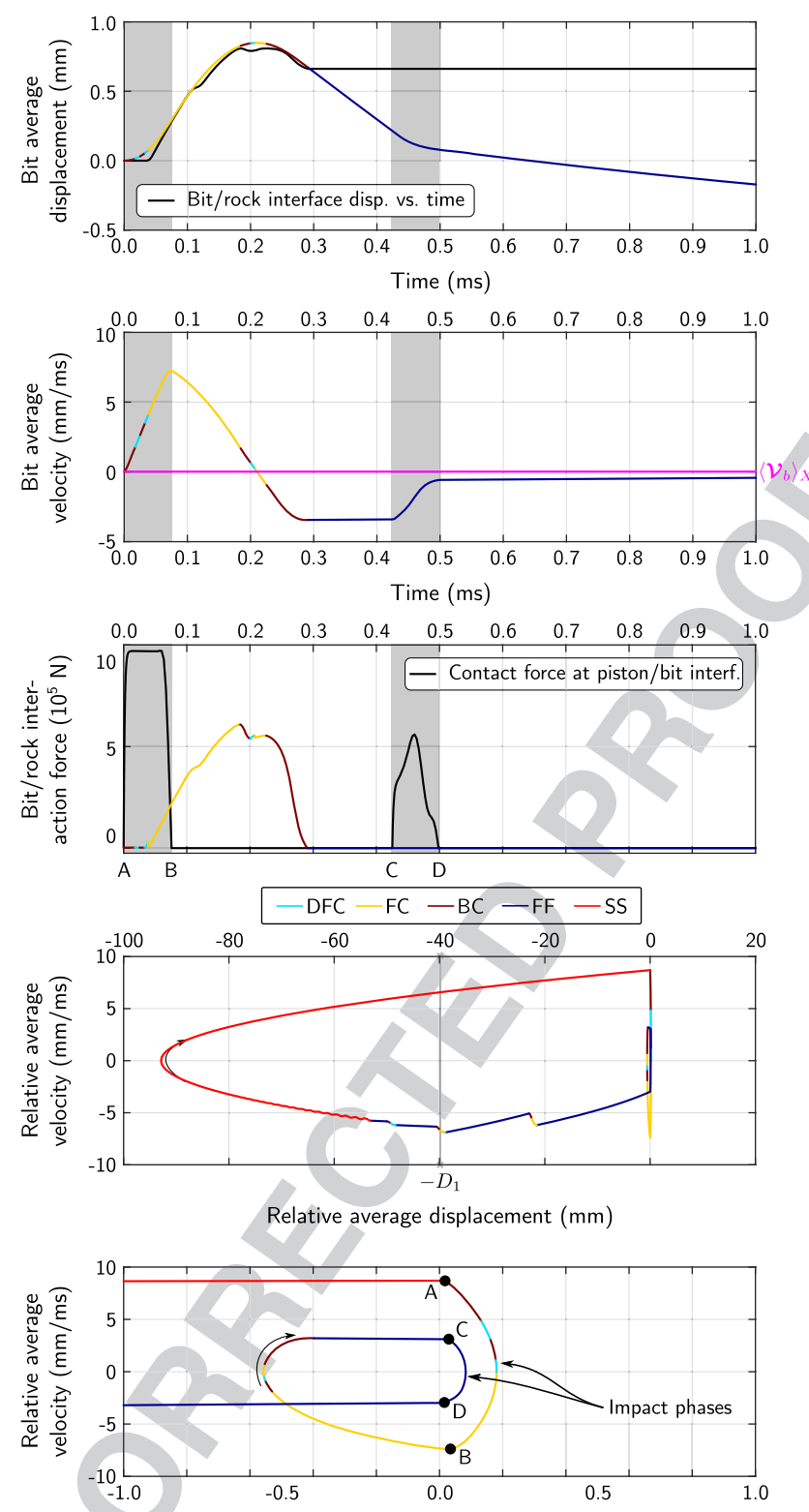

Figure 8. Period-1 solution corresponding to the reference configuration. Time series in the post-activation time range and phase portraits of the relative average motion. The gray shadings correspond to periods of closed contact at the piston/bit interface. DFC, dissipative forward contact; FC, forward contact; BC, backward contact; FF, free flight; SS, standstill.

and the pressure law parameters $F_{0}$ and $D_{1}$ under the constraint of keeping the power scale given in (14) equal to that of the reference configuration. The results reported in Figure 9 are based on the double averaging of the bit velocity, namely

$$
\begin{aligned}
\left\langle\mathcal{V}_{b}\right\rangle_{X, t} & =\frac{1}{T} \int_{t_{0}}^{t_{0}+T}\left\langle\mathcal{V}_{b}\right\rangle_{X}(t) \mathrm{d} t \\
& =\frac{1}{T}\left(\left\langle\mathcal{U}_{b}\right\rangle_{X}\left(t_{0}+T\right)-\left\langle\mathcal{U}_{b}\right\rangle_{X}\left(t_{0}\right)\right),
\end{aligned}
$$

where the time constants have arbitrarily been set to $\left(t_{0}, T\right)=(1 \mathrm{~s}, 3 \mathrm{~s})$, and on the initialization of the model in a generalized FF configuration with $\left(\left\langle\mathcal{V}_{p}\right\rangle_{X}, g_{0}, \mathcal{P}_{u}\right)=\left(10 \mathrm{~mm} / \mathrm{ms}, 10^{-2} \mathrm{~mm}\right.$, $\left.10^{-2} \mathrm{~mm}\right)$. 

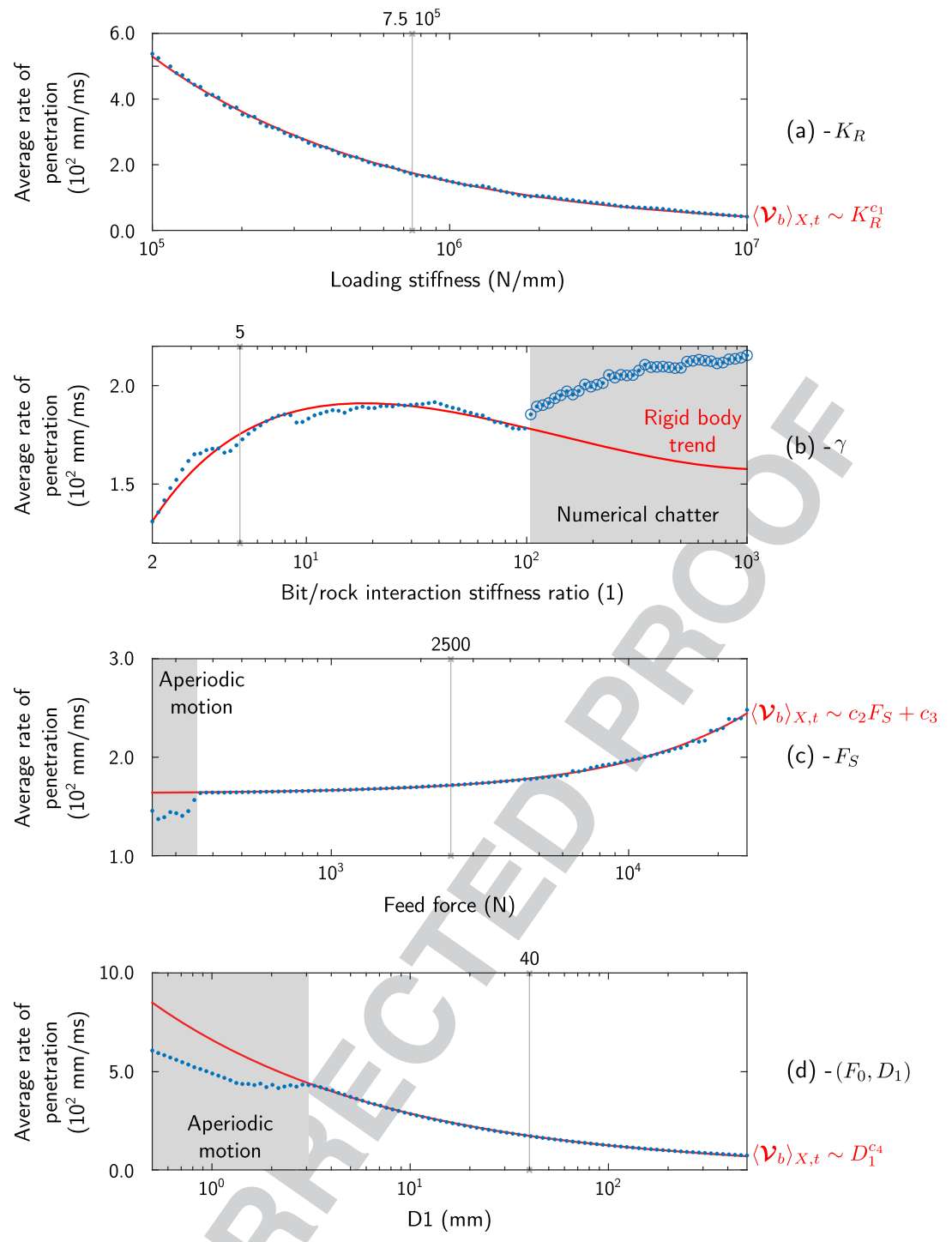

Figure 9. Influence of the model parameters on the predicted average rate of penetration, around the reference configuration. Parameters of the pressure law $\left(F_{0}\right.$ and $\left.D_{1}\right)$ are varied in such a way that the power delivered to the piston remains constant, from a scaling perspective.

Plot (a) shows the influence of the loading stiffness $K_{R}$ on the average rate of penetration; it monotonically decreases as the stiffness increases. A fitting analysis reveals that the power law relating the two quantities is close to the inverse square-root argument, $c_{1} \simeq-0.55$, which is in line with the analysis of the drilling cycle for a rigid bit [31], and follows from the two-phase nature of the stationary motion and the limited elastic effects on the response at the bit/rock interface. This trend illustrates the difficulty of drilling hard rock at important depths, because the magnitude of $K_{R}$, for a given rock, is expected to increase with the confining pressure that itself increases, on average, with the drilling depth.

The influence of unloading parameter $\gamma$ is displayed in plot (b). Starting from $\gamma=2$, the average rate of penetration is seen to increase, on average, with $\gamma$, until a global maximum is reached around $\gamma=40$. Local maxima are observed on the way to the global one; they can be attributed to a change of the number of drilling cycles along the stationary response. Upon further increasing $\gamma$, the average rate of penetration decreases then starts to increase, above $\gamma=100$. This increase reflects the numerical chatter taking place at the bit/rock interface, because of the important difference of 
the stiffness for the loading and unloading modes. Such an increase does not take place for a rigid bit [32], as the drilling process is expected to become insensitive to the value of $\gamma$ when it is large.

In plot (c), the sensitivity of the average rate of penetration to the feed force is illustrated. A linear dependence is revealed by the parametric analysis, provided the feed force is above $F_{S} \simeq 350 \mathrm{~N}$. Below this threshold, forward simulations do not converge to a stationary response. Aperiodic responses are observed whose pattern does not correspond to the two-phase nature of the stationary response studied in Section 4.3; percussive activations take place during any drilling mode, given that limited feed forces allow for large-amplitude FF phases. No sweet spot is observed around the reference configuration. As far as the authors know, there is no explanation to the existence of such optima. Attributing this absence to a specific aspect of the model would thus be pure speculation. Arguments related to the cleaning of the borehole, the simplification of the drilling apparatus geometry, or the effect of the feed force on the bit/rock interaction could well be invoked, as well as the nonexistence of such an optimum at the reference configuration.

The influence of the frequency of the percussive activation is shown in plot (d). Simulation results show that scaling laws (15) are verified as long as the model reaches a stationary long-term motion [32]. In that setting, the rate of penetration is seen to scale as a power law of $D_{1}$, with $\left\langle\mathcal{V}_{b}\right\rangle_{X, t} \sim D_{1}^{c_{4}}$ and $c_{4} \simeq-0.36$. Approximating the power exponent by $c_{4} \simeq-1 / 3$, the average rate of penetration is thus observed to scale with the square root of the percussive activation frequency and the inverse of the piston impact velocity

$$
\left\langle\mathcal{V}_{b}\right\rangle_{X, t} \sim \frac{1}{\sqrt{T_{P}^{*}}}, \quad\left\langle\mathcal{V}_{b}\right\rangle_{X, t} \sim \frac{1}{V_{P}^{*}} .
$$

At high percussive frequencies $\left(D_{1}<3 \mathrm{~mm}\right)$, simulations do not converge to a stationary motion and aperiodic responses are observed. Scaling laws no longer apply in this range of parameters as can be observed from the deviations of the numerical results from the scaling trend; nevertheless, the average rate of penetration still increases, on average, with the decrease of $D_{1}$.

The trends predicted by the model show that the most significant improvement in the rate of penetration can be achieved by increasing the frequency of percussive activation by one or two orders of magnitude. Such an increase of frequency seems difficult to achieve with a fluid-driven hammer. This suggests, under all necessary reserves about the model validity, that investigating alternative technologies driving the hammering process in unexploited nominal functioning ranges could provide a valuable response to the challenges associated with the drilling of hard rock at large depths.

\section{CONCLUSIONS}

It is our contention that percussive drilling should be studied as a dynamical process and its performance assessed from the process long-term response; this departs from the existing body of literature that assumes a specific type of stationary motion to analyze drilling performance. An integrated model of the drilling process, coupling the underlying (sub)processes, is therefore required.

Following that line of thinking, this article presents a novel dynamical model to assess the performance of DTH percussive drilling on the basis of its average long-term response. To represent the process dynamics along multiple percussive activations, the model couples a pressure law that drives the hammer piston to its elastic representation and that of the drill bit, the interaction of the latter with the rock massif being modeled using a generalized bilinear interaction law. The elastic modeling of the piston and the bit is required to properly capture their collisions during the percussive activations, even though for-the interaction of DTH drill bits with hard rock is typically dominated by rigid body dynamics (this is not the case for TH percussive drilling). The model does not account for the angular dynamics of the drilling system nor does it consider the cleaning of the borehole or the tool wear. However, these could well be included in a more advanced dynamical model, which could also consider more sophisticated representations of the included (sub)processes. The presented model should thus be viewed as a basis to build upon to study the performance of percussive drilling. 
The paper details the formulation of the original model as well as its computational version that is based on a spatial semidiscretization using the finite element method combined with an event-driven time integration procedure. The long-term response of the computational model is then studied for a reference configuration corresponding to a $3 \frac{1}{4}$-in hammer, as well as the influence of the bit/rock interaction parameters, the feed force, and the percussive activation parameters on the average rate of penetration.

At the reference configuration, it is observed that forward simulations converge to a stationary response that comprises two phases of motion. The first one, corresponding to a succession of drilling cycles and FF phases where the bit is off the hole bottom, follows from the percussive activation. The second one, where the bit converges to rest on the rock surface, then follows until the next activation takes place. Penetration is achieved during the first phase but not during the second. For the considered configuration, simulations show that two distinct impact phases take place at the piston/bit interface; this rules out the possibility of a comprehensive study of the drilling process by a model of the drifting-oscillator type that would not represent the piston dynamics.

Parametric analyses around the reference configuration reveal that a significant improvement of the rate of penetration is most likely $\rangle_{\wedge}$ achieved by a drastic increase of the frequency of percussive activation rather than by increasing the feed force. This, in turn, prompts for the exploration of new activation technologies that can deliver higher impact frequencies at lower impact velocities. They also show that the influence of the bit/rock interaction parameters is in accordance with expectations; namely, the rate of penetration monotonically decreases with the difficulty to penetrate the rock medium. The existence of a sweet spot, with respect to the feed force, is not revealed around the reference configuration. It is, however, premature to attribute this absence to a missing feature in the proposed model, as such regimes could well be found in other parametric ranges.

To the knowledge of the authors, there is no perfect numerical approach to handle the strain and velocity discontinuities following impacts in 1D elasticity. The proposed computational approach, which is based on a semidiscretization via the finite element method, requires the introduction of artificial damping to stabilize the solution. The calibration of such a numerical addition can sometimes prove difficult. Alternative discretization procedures that can cope with the piecewise-defined nature of the model should thus be explored to reduce the sensitivity of the computational model to numerical factors.

\section{ACKNOWLEDGEMENTS}

A. $\mathrm{B}_{2}$ acknowledges the partial support from the Graduate School of the University of Minnesota (Doctoral Dissertation Fellowship), CSIRO, and Itasca CG. E. D also acknowledges the partial support from the T.W. Bennett chair.

\section{REFERENCES}

1. Bruno MS. Fundamental research on percussion drilling: improved rock mechanics analysis, advanced simulation technology, and full-scale laboratory investigations. Technical Report, Terralog Technologies Inc., 2005.

2. Hustrulid W, Fairhurst C. Theoretical and experimental study of percussive drilling of rock - part I - theory of percussive drilling. International Journal of Rock Mechanics and Mining Sciences and Geomechanics Abstracts $1971 ; 8(4): 311-333$.

3. Han G, Bruno M, Lao K. Percussion drilling in oil industry: review and rock failure modeling. AADE 2005 National Technical Conference and Exhibition, AADE-05-NTCE-59, Houston, Texas, U.S.A., 2005; 1-10.

4. Amjad M. Control of ITH percussive longhole driling in hard rock. Ph.D. Thesis, McGill University, Montreal, Quebec, Canada, 1996.

5. Lundberg B. Energy transfer in percussive rock destruction-I: comparison of percussive methods. International Journal of Rock Mechanics and Mining Sciences and Geomechanics Abstracts 1973; 10(5):381-399.

6. Lundberg B. Energy-transfer in percussive rock destruction-II: supplement on hammer drilling. International Journal of Rock Mechanics and Mining Sciences and Geomechanics Abstracts 1973; 10(5):401-419. 
7. Simon R. Transfer of the stress wave energy in the drill steel of a percussive drill to the rock. International Journal of Rock Mechanics and Mining Sciences and Geomechanics Abstracts 1964; 1(3):397-411.

8. Lundberg B. Computer modelling and simulation of percussive drilling of rock. In Comprehensive Rock Engineering, Hudson JA (ed.). Pergamon, 1993.

9. Chiang LE, Elías DA. A 3D FEM methodology for simulating the impact in rock-drilling hammers. International Journal of Rock Mechanics and Mining Sciences 2008; 45(5):701-711.

10. Chiang LE, Elias DA. Modeling impact in down-the-hole rock drilling. International Journal of Rock Mechanics and Mining Sciences 2000; 37(4):599-613.

11. Lundberg B, Okrouhlik M. Influence of 3D effects on the efficiency of percussive rock drilling. International Journal of Impact Engineering 2001; 25(4):345-360.

12. Lundberg B, Okrouhlik M. Efficiency of a percussive rock drilling process with consideration of wave energy radiation into the rock. International Journal of Impact Engineering 2006; 32(10):1573-1583.

13. Ajibose OK, Wiercigroch M, Pavlovskaia E, Akisnaya AK. Global and local dynamics of drifting oscillator for different contact force models. International Journal of Non-Linear Mechanics 2010; 45(9):850-858.

14. Ajibose OK, Wiercigroch M, Pavlovskaia E, Akisanya AR, Karolyi G. Drifting impact oscillator with a new model of the progression phase. Journal of Applied Mechanics - Transactions of the ASME 2012; 79(6).

15. Karlsson LG, Lundberg B, Sundin KG. Experimental study of a percussive process for rock fragmentation. International Journal of Rock Mechanics and Mining Sciences and Geomechanics Abstracts 1989; 26(1):45-50.

16. Depouhon A, Detournay E, Denoël V. Event-driven integration of linear structural dynamics models under unilateral elastic constraints. Computer Methods in Applied Mechanics and Engineering 2014; 276:312-340.

17. Chiang LE, Stamm EB. Design optimization of valveless DTH pneumatic hammers by a weighted pseudo-gradient search method. Journal of Mechanical Design 1998; 120(4):687-694.

18. Wang SY, Sloan SW, Liu HY, Tang CA. Numerical simulation of the rock fragmentation process induced by two drill bits subjected to static and dynamic (impact) loading. Rock Mechanics and Rock Engineering 2011; 44(3): 317-332.

19. Saksala T. 3D numerical modelling of bit-rock fracture mechanisms in percussive drilling with a multiple-button bit. International Journal for Numerical and Analytical Methods in Geomechanics 2013; 37(3):309-324.

20. Géradin M, Rixen D. Mechanical Vibrations: Theory and Application to Structural Dynamics. Wiley, 1997.

21. Graff KF. Wave Motion in Elastic Solids, Dover Books on Engineering Series. Dover Publications, 1975.

22. Kolsky H. Stress Waves in Solids, Dover phoenix editions. Dover Publications, 2003.

23. Doyen D, Ern A, Piperno S. Time-integration schemes for the finite element dynamic Signorini problem. SIAM Journal on Scientific Computing 2011; 33(1):223-249.

24. Izquierdo LE, Chiang LE. A methodology for estimation of the specific rock energy index using corrected down-thehole drill monitoring data. Mining Technology 2004; 113(4):225-236.

25. Haimson B. High velocity, low velocity and static penetration characteristics in Tennessee marble. Master's Thesis, University of Minnesota, 1965.

26. Stephenson BR. Measurement of dynamic force-penetration characteristics in Indiana limestone. Master's Thesis, University of Minnesota, 1963.

27. Ajibose OK. Nonlinear dynamics and contact fracture mechanics of high frequency percussive drilling. Ph.D. Thesis, University of Aberdeen, 2009.

28. Saksala T. Numerical modelling of bit-rock fracture mechanisms in percussive drilling with a continuum approach. International Journal for Numerical and Analytical Methods in Geomechanics 2011; 35(13):1483-1505.

29. Hustrulid W, Fairhurst C. A theoretical and experimental study of the percussive drilling of rock part III-experimental verification of the mathematical theory. International Journal of Rock Mechanics and Mining Sciences and Geomechanics Abstracts 1972; 9(3):417-418.

30. Saksala T, Gomon D, Hokka M, Kuokkala VT. Numerical and experimental study of percussive drilling with a triple-button bit on Kuru granite. International Journal of Impact Engineering 2014; 72:56-66.

31. Depouhon A, Denoël V, Detournay E. A drifting impact oscillator with periodic impulsive loading: application to percussive drilling. Physica D 2013; 258(0):1-10.

32. Depouhon A. Integrated dynamical models for down-the-hole percussive drilling. Ph.D. Thesis, Université de Liège, University of Minnesota, 2014.

33. Hughes TJR. The Finite Element Method: Linear Static and Dynamic Finite Element Analysis. Dover, 1987.

34. Wriggers P. Computational Contact Mechanics. John Wiley and Sons, Ltd, 2006.

35. Pfeiffer F, Glocker C. Multibody Dynamics With Unilateral Contacts, Vol. 9. Wiley-VCH, 1996.

36. Acary V, Brogliato B. Numerical Methods for Nonsmooth Dynamical Systems. Applications in Mechanics and Electronics. Springer-Verlag: Berlin Heidelberg, 2008.

37. Depouhon A, Detournay E, Denoël V. Accuracy of one-step integration schemes for damped/forced linear structural dynamics. International Journal for Numerical Methods in Engineering 2014; 99(5):333-353.

38. Banks J, Henshaw W. Upwind schemes for the wave equation in second-order form. Journal of Computational Physics 2012; 231(17):5854-5889.

39. Personal communications with J. Aros, R\&D Manager, DrillCo Tools S.A. 


\section{Journal: International Journal for Numerical and Analytical Methods in Geome- chanics}

\section{Article: nag_2344}

Dear Author,

During the copyediting of your paper, the following queries arose. Please respond to these by annotating your proofs with the necessary changes/additions.

- If you intend to annotate your proof electronically, please refer to the E-annotation guidelines.

- If you intend to annotate your proof by means of hard-copy mark-up, please refer to the proof mark-up symbols guidelines. If manually writing corrections on your proof and returning it by fax, do not write too close to the edge of the paper. Please remember that illegible mark-ups may delay publication.

Whether you opt for hard-copy or electronic annotation of your proofs, we recommend that you provide additional clarification of answers to queries by entering your answers on the query sheet, in addition to the text mark-up.

\begin{tabular}{|c|l|c|}
\hline Query No. & \multicolumn{1}{|c|}{ Query } & Remark \\
\hline Q1 & $\begin{array}{l}\text { AUTHOR: Figures 1 and 3-9 are recommended for color } \\
\text { online and in print. Please check. }\end{array}$ \\
\hline Q2 & $\begin{array}{l}\text { AUTHOR: Please provide city location and page range for } \\
\text { Reference 8. }\end{array}$ & \\
\hline Q3 & AUTHOR: Please provide page range for Reference 14. \\
\hline Q4 & $\begin{array}{l}\text { AUTHOR: Please provide city location for References 20-22 } \\
\text { and 33-35. }\end{array}$ \\
\hline
\end{tabular}


Required software to e-Annotate PDFs: Adobe Acrobat Professional or Adobe Reader (version 7.0 or above). (Note that this document uses screenshots from Adobe Reader $\mathrm{X}$ )

The latest version of Acrobat Reader can be downloaded for free at: http://get.adobe.com/uk/reader/

Once you have Acrobat Reader open on your computer, click on the Comment tab at the right of the toolbar:

닙

This will open up a panel down the right side of the document. The majority of tools you will use for annotating your proof will be in the Annotations section, pictured opposite. We've picked out some of these tools below:

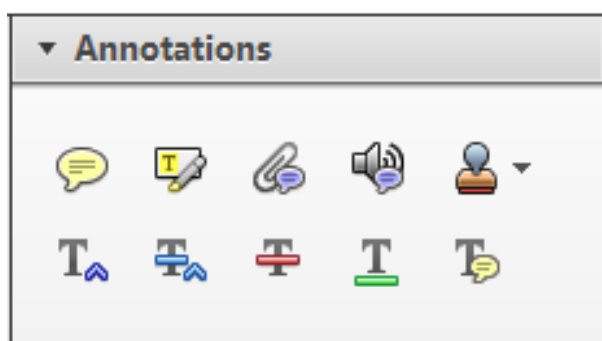

1. Replace (Ins) Tool - for replacing text.

Strikes a line through text and opens up a text box where replacement text can be entered.

How to use it

- Highlight a word or sentence.

- Click on the Replace (Ins) icon in the Annotations section.

- Type the replacement text into the blue box that appears.

Idard tramework for the analysis of $\mathrm{m}$ icy-Nevertheless, it also led to exog،

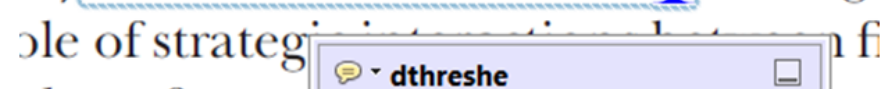
\begin{tabular}{l|l|l} 
aber of comp & 08/06/2011 15:58:17 & 0
\end{tabular} is that the $\mathrm{s} 1 \overline{\text {, which led }}$ of nain compo: be level, are exc nc

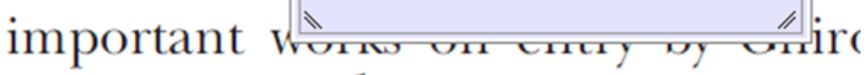
M heneferth) ${ }^{1}$ we anen the "hlarl $\mathrm{h}$

3. Add note to text Tool - for highlighting a section to be changed to bold or italic.

T Highlights text in yellow and opens up a text box where comments can be entered.

How to use it

- Highlight the relevant section of text.

- Click on the Add note to text icon in the Annotations section.

- Type instruction on what should be changed regarding the text into the yellow box that appears.

namic responses of mark ups ent with the VAR evidence

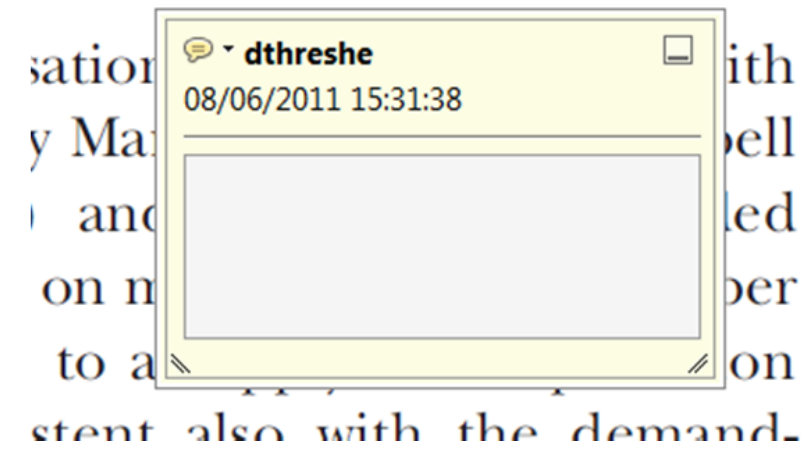

2. Strikethrough (Del) Tool - for deleting text.

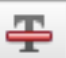

Strikes a red line through text that is to be deleted.

How to use it

- Highlight a word or sentence.

- Click on the Strikethrough (Del) icon in the Annotations section.

there is no room tor extra prohts al s ups are zero and the number of ret) values are not determined by Blanchard and Kiyotaki (1987), sfect competition in general equilil ts of aggregate demand and supply lassical framework assuming monol sen an evorenous number of firms

4. Add sticky note Tool - for making notes at specific points in the text.

Marks a point in the proof where a comment needs to be highlighted.

How to use it

- Click on the Add sticky note icon in the Annotations section.

- Click at the point in the proof where the comment should be inserted.

- Type the comment into the yellow box that appears.

iaisu airu suppiy sisuks. hivsl ui

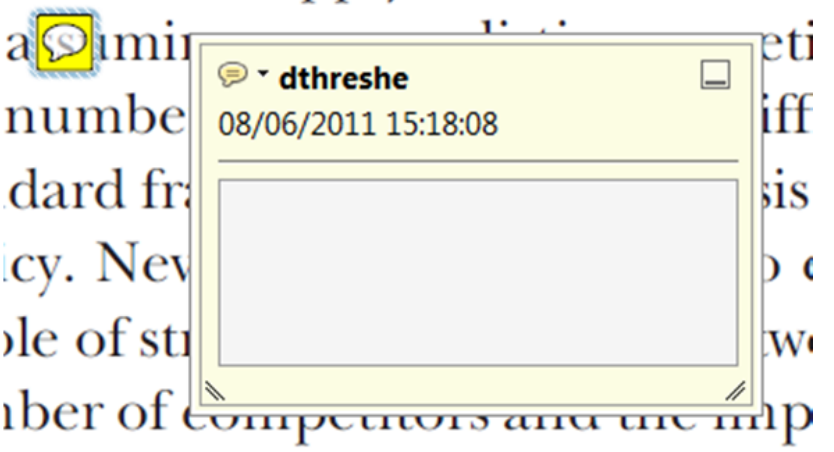

is that the structure of the sectc. 
5. Attach File Tool - for inserting large amounts of text or replacement figures.

Inserts an icon linking to the attached file in the appropriate pace in the text.

How to use it

- $\quad$ Click on the Attach File icon in the Annotations section.

- Click on the proof to where you'd like the attached file to be linked.

- Select the file to be attached from your computer or network.

- Select the colour and type of icon that will appear in the proof. Click OK.

E N D

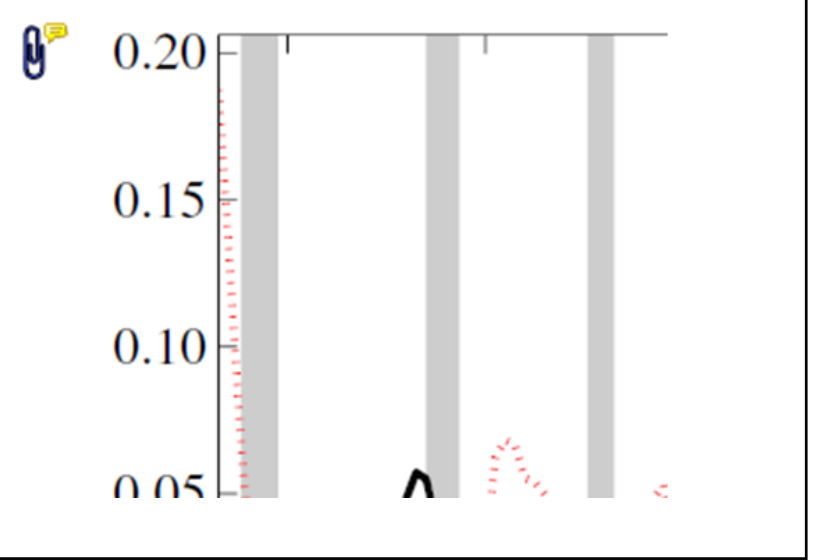

6. Add stamp Tool - for approving a proof if no corrections are required.

- Inserts a selected stamp onto an appropriate place in the proof.

\section{How to use it}

- $\quad$ Click on the Add stamp icon in the Annotations section.

- Select the stamp you want to use. (The Approved stamp is usually available directly in the menu that appears).

- Click on the proof where you'd like the stamp to appear. (Where a proof is to be approved as it is, this would normally be on the first page).

)t the Dusiness cycie, starting with the on perfect competition, constant ret

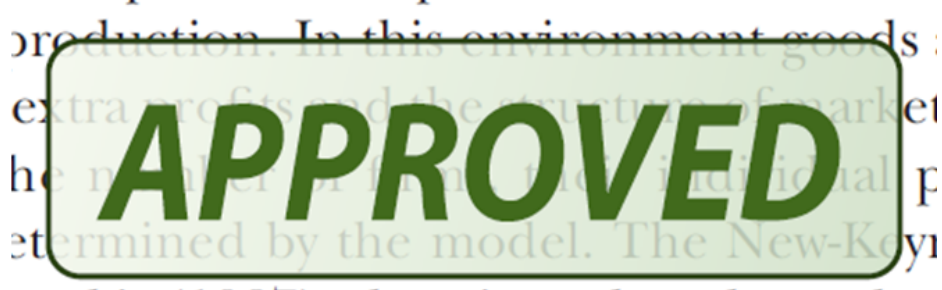
otaki (1987), has introduced produc general equilibrium models with nomin:

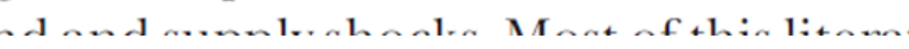

- Drawing Markups

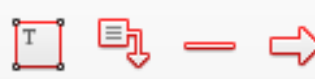

$0 \bigcirc \sqrt{6} \otimes$

\section{How to use it}

- Click on one of the shapes in the Drawing Markups section.

- Click on the proof at the relevant point and draw the selected shape with the cursor.

- To add a comment to the drawn shape, move the cursor over the shape until an arrowhead appears.

- Double click on the shape and type any text in the red box that appears.
7. Drawing Markups Tools - for drawing shapes, lines and freeform annotations on proofs and commenting on these marks.

Allows shapes, lines and freeform annotations to be drawn on proofs and for comment to be made on these marks.

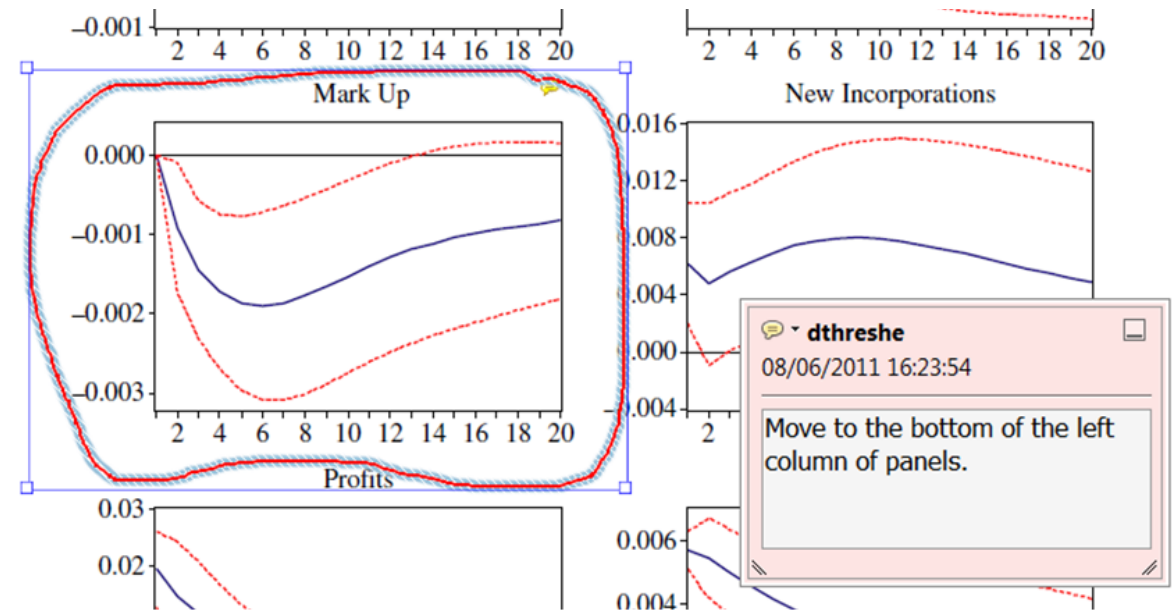

For further information on how to annotate proofs, click on the Help menu to reveal a list of further options:

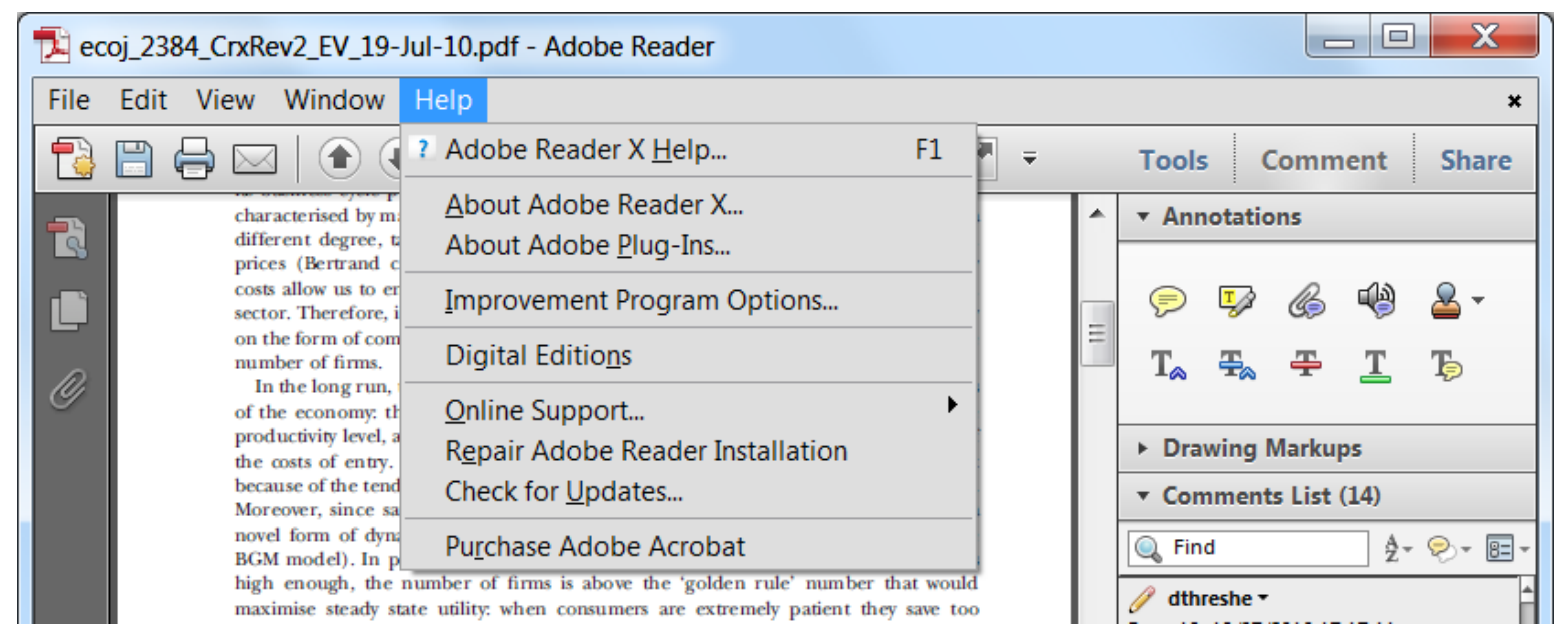

